\title{
RESEARCH
}

Open Access

\section{Baicalin ameliorates neuroinflammation- induced depressive-like behavior through inhibition of toll-like receptor 4 expression via the PI3K/AKT/FoxO1 pathway}

Li-Ting Guo', Si-Qi Wang ${ }^{1}$, Jing Su${ }^{1}$, Li-Xing Xu' ${ }^{1}$, Zhou-Ye Ji', Ru-Yi Zhang ${ }^{1}$, Qin-Wen Zhao ${ }^{1}$, Zhan-Qiang Ma ${ }^{1 *}$, Xue-Yang Deng ${ }^{1 *}$ and Shi-Ping Ma ${ }^{1,2^{*}}$ (D)

\begin{abstract}
Background: Baicalin, which is isolated from Radix Scutellariae, possesses strong biological activities including an anti-inflammation property. Recent studies have shown that the anti-inflammatory effect of baicalin is linked to toll-like receptor 4 (TLR4), which participates in pathological changes of central nervous system diseases such as depression. In this study, we explored whether baicalin could produce antidepressant effects via regulation of TLR4 signaling in mice and attempted to elucidate the underlying mechanisms.

Methods: A chronic unpredictable mild stress (CUMS) mice model was performed to explore whether baicalin could produce antidepressant effects via the inhibition of neuroinflammation. To clarify the role of TLR4 in the anti-neuroinflammatory efficacy of baicalin, a lipopolysaccharide (LPS) was employed in mice to specially activate TLR4 and the behavioral changes were determined. Furthermore, we used LY294002 to examine the molecular mechanisms of baicalin in regulating the expression of TLR4 in vivo and in vitro using western blot, ELISA kits, and immunostaining. In the in vitro tests, the BV2 microglia cell lines and primary microglia cultures were pretreated with baicalin and LY292002 for $1 \mathrm{~h}$ and then stimulated $24 \mathrm{~h}$ with LPS. The primary microglial cells were transfected with the forkhead transcription factor forkhead box protein $\mathrm{O} 1$ (FoxO1)-specific siRNA for $5 \mathrm{~h}$ and then co-stimulated with baicalin and LPS to investigate whether FoxO1 participated in the effect of baicalin on TLR4 expression.

*Correspondence: mazhanqiang0427@163.com; dxy@cpu.edu.cn; cpu435@163.com

'Department of Pharmacology of Chinese Materia Medica, China

Pharmaceutical University, Tongjiaxiang 24, Nanjing 210009, Jiangsu, People's

Republic of China

Full list of author information is available at the end of the article

(c) The Author(s). 2019 Open Access This article is distributed under the terms of the Creative Commons Attribution 4.0 International License (http://creativecommons.org/licenses/by/4.0/), which permits unrestricted use, distribution, and reproduction in any medium, provided you give appropriate credit to the original author(s) and the source, provide a link to the Creative Commons license, and indicate if changes were made. The Creative Commons Public Domain Dedication waiver (http://creativecommons.org/publicdomain/zero/1.0/) applies to the data made available in this article, unless otherwise stated. 
(Continued from previous page)

Results: The administration of baicalin (especially $60 \mathrm{mg} / \mathrm{kg}$ ) dramatically ameliorated CUMS-induced depressive-like symptoms; substantially decreased the levels of interleukin-1 beta (IL-1 $\beta$ ), interleukin-6 (IL-6), and tumor necrosis factor alpha (TNF-a) in the hippocampus; and significantly decreased the expression of TLR4. The activation of TLR4 by the LPS triggered neuroinflammation and evoked depressive-like behaviors in mice, which were also alleviated by the treatment with baicalin $(60 \mathrm{mg} / \mathrm{kg}$ ). Furthermore, the application of baicalin significantly increased the phosphorylation of phosphatidylinositol 3-kinase (PI3K), protein kinase B (AKT), and FoxO1. The application of baicalin also promoted FoxO1 nuclear exclusion and contributed to the inhibition of the FoxO1 transactivation potential, which led to the downregulation of the expression of TLR4 in CUMS mice or LPS-treated BV2 cells and primary microglia cells. However, prophylactic treatment of LY294002 abolished the above effects of baicalin. In addition, we found that FoxO1 played a vital role in baicalin by regulating the TLR4 and TLR4-mediating neuroinflammation triggered by the LPS via knocking down the expression of FoxO1 in the primary microglia.

Conclusion: Collectively, these results demonstrate that baicalin ameliorated neuroinflammation-induced depressivelike behaviors through the inhibition of TLR4 expression via the PI3K/AKT/FoxO1 pathway.

Keywords: Depression, Baicalin, Neuroinflammation, TLR4, FoxO1

\section{Background}

Depression is a life-threatening and debilitating mental disorder characterized by melancholy feelings and a lack of interest and motivation. Increasing amounts of data suggest that neuroinflammation is associated with the etiology of depression [1-3]. Preclinical and clinical research has demonstrated that central and peripheral elevation of pro-inflammation cytokines might be contributors to depressive-like behaviors [4]. Preclinical research has shown that pro-inflammation cytokines, including IL-1 $\beta$, IL- 6 , and TNF- $\alpha$, play a vital role in the onset and progression of inflammation, which subsequently induces depressive behaviors [5-7]. It has been previously shown that major depressive disorder (MDD) patients exhibited higher levels of IL- 6 and TNF- $\alpha$ in the blood and cerebrospinal fluid (CSF) $[8,9]$. In addition, the expression of IL-1 $\beta$, IL- 6 , and TNF- $\alpha$ was also increased in postmortem brain samples from suicide victims that suffered from depression [10]. Most psychiatric and neurological diseases are aggravated by stress. Stress-evoked mood disorders are partially related to the dysfunction of the inflammatory system, and compelling evidence indicates that the stress-evoked elevation of inflammatory factors contributes to depression and may even deteriorate it further [3-12].

TLR4 is a pattern recognition receptor expressed in a variety of cells, including macrophages, microglia, astrocytes, and neurons [13], and has regulatory roles in the adrenal response to stress and inflammatory stimuli as well as the brain response to stress $[14,15]$. The expression of TLR4 is not static because it is swiftly regulated in response to pathogens, various cytokines, and environmental stresses [16]. Activation of the TLR4 complex might underlie the pathophysiology of stress-evoked depression. This was shown by repressing stress-elevated TLR4 expression in the rodent brain and inhibiting
TLR4 activity mitigated stress-induced NF- $\kappa B$ activation and attenuation of the upregulation of pro-inflammation cytokines both in the hippocampus and prefrontal cortex $[17,18]$. Although some antidepressants exert effects by inhibiting the expression of TLR4, the exact mechanism of regulating TLR4 expression remains unclear.

Baicalin ( $\beta$-D-glucopyranosiduronic acid, 5, 6-dihydroxy4-oxo-2-phenyl-4H-1-benzopyran-7-yl, $M=446.36$, Fig. 1a), which is a lipophilic flavonoid glycoside $(\log P=1.27)$ [19] isolated from Radix Scutellariae, possesses strong biological activities including anti-inflammatory, antioxidant, and antiapoptotic effects [20]. Intriguingly, the current research has shown that baicalin exerts neuroprotective and anti-neuroinflammatory effects by regulating the activation of $5^{\prime}$ adenosine monophosphate-activated protein kinase (AMPK) [21] and the phosphatidylinositol 3-kinase (PI3K)/ protein kinase $\mathrm{B}(\mathrm{AKT})$ pathway $[10,22]$. FoxO1, which is a member of the FoxO family, has transactivation activity by binding to multiple enhancer-like elements within the TLR4 gene [23]. The transcriptional activity of FoxO1 is mediated by the PI3K/AKT pathway and other signal pathways [24]. Importantly, recent findings revealed that baicalin can be used to treat a variety of inflammatory diseases through the downregulation of TLR4 expression including LPS-induced liver inflammation [25], experimental periodontitis [26], acute pancreatitis [27], and experimental colitis [28]. Collectively, we hypothesized that baicalin might ameliorate neuroinflammation-induced depressive-like behaviors via the regulation of TLR4 expression. To investigate this hypothesis, we created chronic and acute stress tests to examine the activation of TLR4 and the levels of IL-1 $\beta$, IL- 6 , and TNF- $\alpha$ in the hippocampus of depressive mice and then further investigated the possible molecular and cellular mechanisms underlying the antidepressive effects of baicalin in BV2 line cells and primary microglia cells. 


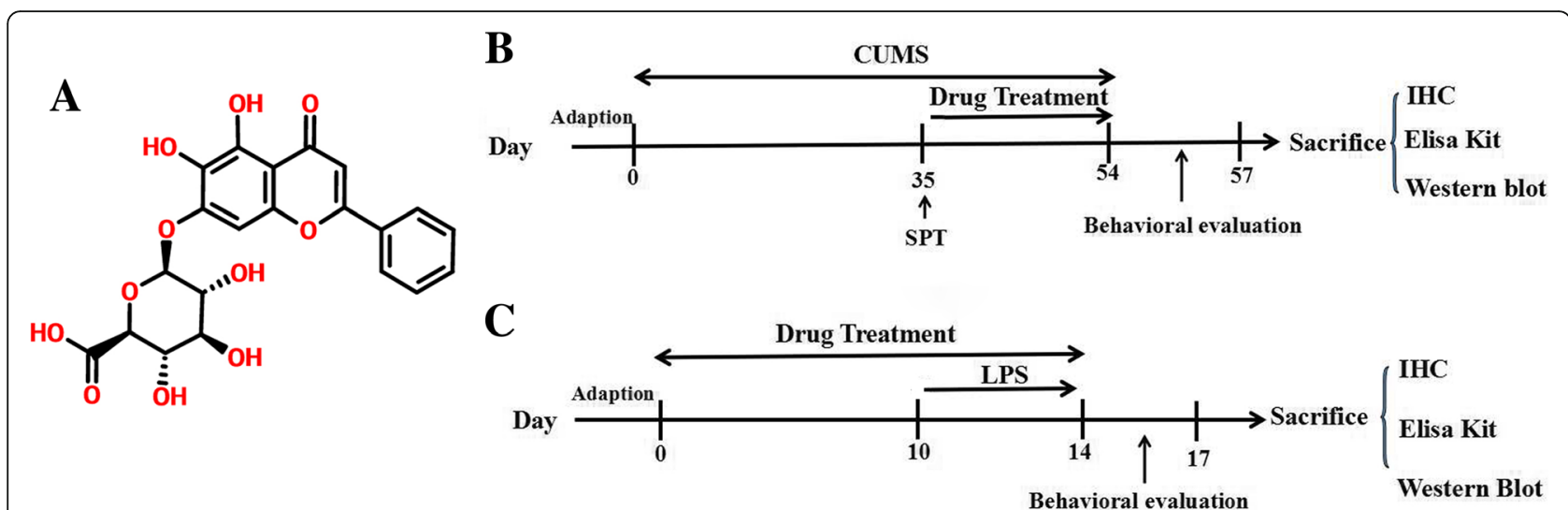

Fig. 1 a The structural formula of baicalin. b A schematic representation of the CUMS procedure and treatments in mice. c A schematic representation of the LPS procedure and treatments in mice

\section{Methods}

\section{Drugs and reagents}

Baicalin (purity $\geq 98 \%$ ) was obtained from Xi'an Kai Lai Biological Engineering Co., Ltd. (Xi'an, China). Fluoxetine hydrochloride (Flu) was purchased from Changzhou Siyao Pharmaceuticals Co., Ltd. (Changzhou, China). Lipopolysaccharide (LPS), 4,6-diamidino-2-phenylindole (DAPI), and compound $\mathrm{C}$ were bought from Sigma-Aldrich Co (St. Louis, USA). TAK-242 (a TLR4 antagonist) and LY294002 (a PI3K inhibitor) were products purchased from Apex Bio (Houston, USA). Poly-D-lysine was obtained from Sigma (MO, USA). Interleukin-1 $\beta$ (IL-1 $\beta$ ), Interleukin-6 (IL-6), and tumor necrosis factor $\alpha$ (TNF- $\alpha$ ) enzyme-linked immunosorbent assay (ELISA) kits were supplied by Elabscience Biotechnology Co., Ltd. (Wuhan, China). The antibodies were obtained from the cited commercial sources: anti-p-PI3K (\#4228), anti-PI3K (\#4292), anti-p-Akt (Ser473, \#9271), anti-Akt (\#9272), anti- $\beta$-actin (\#4967), anti-PCNA (\#13110), anti-p-AMPK (\#2531), and anti-AMPK (\#2603) were from Cell Signaling Technology (Beverly, MA, USA); anti-PTEN (sc-7974) and anti-p-PTEN (sc-377573) were from Santa Cruz Biotechnology (Santa Cruz, CA); anti-TLR4 (AF7017), anti-pGSK3 $\beta$ (AF2016), and anti-GSK3 $\beta$ (AF7814) were from Affinity Biosciences (Changzhou, China); and anti-FoxO1 (ab52857), anti-p-FoxO1 (Ser 256, ab131339), and goat anti-rabbit IgG H\&L (Alexa Fluor ${ }^{\circledR} 488$, ab150077) were from Abcam (Cambridge, MA, USA).

\section{Animals}

The experiments were performed on male ICR mice (18-22 g), which were obtained from the Experimental Animal Center in Jiangsu Province (Nanjing, China) and maintained under standard laboratory conditions. The mice were randomly housed in cages under a 12-h light/ dark cycle (lights on at 7:00 a.m.), 60\% humidity, and a temperature of $24 \pm 1^{\circ} \mathrm{C}$ with free access to water and food. All of the procedures were strictly performed in accordance with the Provision and General Recommendation of the Chinese Experimental Animals Administration Legislation and were approved by the Science and Technology Department of Jiangsu Province.

\section{CUMS protocol and treatments}

The chronic unpredictable mild stress (CUMS) procedure was performed as described earlier with minor modifications [29-31]. After adaptation, mice in the CUMS groups were subjected to different stressors for 8 weeks as shown in Table 1. Two different stressors were applied each day, and the sequence of stressors changed every 3 days. Non-stressed mice were kept in undisturbed cages. The schematic of the experimental procedure is shown in Fig. 1b. After 5 weeks of the CUMS procedure, the sucrose preference test was performed to measure whether the CUMS model was successful.

Mice exhibiting anhedonia behavior, which is a principal symptom of the CUMS model [31, 32], were

Table 1 The stressors of CUMS protocol

\begin{tabular}{ll}
\hline Stressor & Duration \\
\hline Water deprivation & $12 \mathrm{~h}$ \\
Soiled cage & $12 \mathrm{~h}$ \\
Food deprivation & $12 \mathrm{~h}$ \\
Cage title $\left(45^{\circ}\right)$ & $12 \mathrm{~h}$ \\
Empty bottle & $12 \mathrm{~h}$ \\
Overnight illumination & $12 \mathrm{~h}$ \\
Physical restrain & $1 \mathrm{~h}$ \\
Cage shaking & $10 \mathrm{~min}$ \\
Tail nipping & $10 \mathrm{~min}$ \\
Overhang & $10 \mathrm{~min}$ \\
Noise & $10 \mathrm{~min}$ \\
\hline
\end{tabular}


randomly assigned to four groups ( $n=15$ in each group): CUMS group, CUMS+Flu (fluoxetine, $20 \mathrm{mg} / \mathrm{kg}$ [12], i.g.) group, CUMS+BA30 (baicalin, $30 \mathrm{mg} / \mathrm{kg}$, i.g.) group, and CUMS+BA60 (baicalin, $60 \mathrm{mg} / \mathrm{kg}$, i.g.) group. Non-stressed mice were randomly divided into two groups ( $n=15$ in each group): the control group and the control+BA60 (baicalin, $60 \mathrm{mg} / \mathrm{kg}$, i.g.). The doses of baicalin were selected based on earlier reports [33, 34]. From the sixth week, baicalin or Flu was intragastrically administered once daily for the subsequent 3 weeks and chronic treatments and CUMS stimulus were continued throughout the treatment period. Mice in the control group and the CUMS group were given an equal volume of double distilled water.

In addition, another CUMS protocol was designed to explore the further mechanism of baicalin. The mice were administered the aforementioned CUMS procedure and assigned to four groups ( $n=15$ in each group): control group (non-stress), CUMS group, CUMS+BA60 (baicalin, $60 \mathrm{mg} / \mathrm{kg}$, i.g.), and CUMS+LY+BA60 (LY294002, $6 \mu \mathrm{g}$, intranasally; baicalin, $60 \mathrm{mg} / \mathrm{kg}$, i.g.). LY294002 was administered intranasally to mice $1 \mathrm{~h}$ before the administration of baicalin. The administration method and dose of LY294002 were taken from previous reports [35, 36]. After a 3-week treatment, the behavioral tests were performed and the animals were sacrificed after behavioral experiments.

\section{LPS protocol and treatments}

After the adaptation, male mice were randomly allocated into four groups ( $n=12$ in each group): the control group, LPS group, LPS+TAK (TAK-242, $3 \mathrm{mg} / \mathrm{kg}$, i.p.) group, and LPS+BA60 (baicalin, $60 \mathrm{mg} / \mathrm{kg}$, i.g.). TAK-242 or baicalin was administered once daily for 14 consecutive days, and LPS was administered intraperitoneally once a day for the subsequent 5 days from the tenth day as shown in Fig. 1c. The doses of TAK-242 were chosen based on earlier reports [37, 38]. LPS was injected (i.p.) at the dose of $0.83 \mathrm{mg} / \mathrm{kg}$ and according to the previous studies [39-41]. The behavioral tests were performed $12 \mathrm{~h}$ after the last LPS administration.

\section{Behavioral evaluation}

All of the behavioral tests were conducted in parallel in all of the animals. The protocol followed the sequence of sucrose preference test (SPT), open field test (OFT), tail suspension test (TST), and forced swimming test (FST) as previously described [8-10]. This sequence was chosen to expose the animals to the same experimental conditions; however, mice were taken in a random sequence to avoid testing an entire group at once. All of the behavioral tests were conducted blindly with respect to the surgery and drug administration and under conditions of dim light and low noise.

\section{Sucrose preference test}

Anhedonia (as a core symptom of CUMS mice) was monitored using the SPT as previously described [42, 44]. Briefly, the mice were acclimatized to a $1 \%$ sucrose solution $(w / v)$ for $24 \mathrm{~h}$ before the test. After adaptation, the mice were deprived of water and food for $12 \mathrm{~h}$. Then, the mice were housed individually and given two bottles with tap water and a $1 \%$ sucrose solution for $12 \mathrm{~h}$, and the positions of the two bottles were mutually exchanged after $6 \mathrm{~h}$ to avoid side preference. The sucrose preference (SP) value was calculated as: SP $(\%)=$ sucrose intake $(\mathrm{g}) /[\mathrm{su}-$ crose intake $(\mathrm{g})+$ water intake $(\mathrm{g})] \times 100 \%$.

\section{Open field test}

The OFT was generally used to evaluate the locomotor activity, and the test was performed as previously described [34]. Mice were individually placed into the center of an open field apparatus and permitted free exploration for $6 \mathrm{~min}$. After two minutes of adaptation to the apparatus, the number of crossings was recorded during the next $4 \mathrm{~min}$. The device was cleaned with ethanol after each test.

\section{Tail suspension test}

The TST was conducted based on earlier methodology [43]. Each mouse was suspended upside down for $6 \mathrm{~min}$. After $2 \mathrm{~min}$ of adaptation, the immobility durations over the next 4 min were measured.

\section{Forced swimming test}

The FST was conducted as the previously described methodology $[45,46]$. The apparatus was a hyaline cylinder $\left(15 \mathrm{~cm}\right.$ diameter) filled with $22-25^{\circ} \mathrm{C}$ water to a depth of $20 \mathrm{~cm}$. The mice were exposed to a training period of $15 \mathrm{~min}$, and $24 \mathrm{~h}$ later, the mice were individually placed in the water to swim for $6 \mathrm{~min}$ and the immobile time was calculated during the final $4 \mathrm{~min}$.

\section{Immunohistochemistry and Nissl staining}

The mice were deeply anesthetized with $10 \%(w / v)$ chloral hydrate and transcardially perfused with $100 \mathrm{~mL}$ of phosphate buffer saline (PBS, pH 7.3) followed by 50 $\mathrm{mL}$ of $4 \%(w / v)$ paraformaldehyde in PBS [47]. The brains were removed and immersed in 10\% formalin overnight at $4{ }^{\circ} \mathrm{C}$ and then processed for paraffin embedding and sectioned into coronal slices of $6 \mu \mathrm{m}$ [30]. The procedure was performed as previously described [48]. Briefly, the paraffin sections were deparaffinized, rehydrated, and blocked. Subsequently, the sections were incubated with rabbit anti-TLR4 (1:100) overnight for $4{ }^{\circ} \mathrm{C}$. The sections were washed with PBS and incubated with goat anti-rabbit secondary antibody at room temperature for 1 $\mathrm{h}$. The samples were rinsed, and a 3, 3-diaminobenzidine color reaction was performed. After dehydration, the 
sections were transparentized. The images of the hippocampal CA1 region were collected using light microscopy and quantified by Image-Pro Plus 6 (IPP 6).

For Nissl staining [49], the brain slices were dewaxed with xylene and hydrated in a graded series of alcohol. Then, the samples were stained by a Nissl solution for $10 \mathrm{~min}$, washed with distilled water, dehydrated by gradient alcohol, transparentized by xylene, and mounted by neutral balsam. Next, the sections were observed and photographed under a microscope. The numbers of positive cells were quantified in the CA1 hippocampal subfield with IPP 6.

\section{Cell culture and treatments}

The murine BV2 microglial cells were cultured in Dulbecco's modified Eagle's medium (DMEM, Gibco) supplemented with $10 \%$ fetal bovine serum (FBS, Biological Industries) and incubated at $37^{\circ} \mathrm{C}$ in a humidified incubator under $5 \% \mathrm{CO}_{2}$. The cells were pretreated with baicalin $(100 \mu \mathrm{M})$ and LY292002 $(10 \mu \mathrm{M})$ for $1 \mathrm{~h}$ followed by post-incubation with LPS $(1 \mu \mathrm{g} / \mathrm{mL})$ for $24 \mathrm{~h}$. The concentration of baicalin was selected by the MTT assay, and the concentration of LPS and LY294402 was considered according to previously described studies $[50,51]$.

\section{Primary mouse microglial cell culture}

Primary microglia cultures were prepared following standard methods [52, 53] (details in Additional file 1) and cultured in the Dulbecco's modified Eagle's medium nutrient mixture F-12 (DMEM/F12, Gibco) containing $10 \% \mathrm{FBS}$ and antibiotics $(40 \mathrm{U} / \mathrm{mL}$ penicillin and $40 \mu \mathrm{g} /$ $\mathrm{mL}$ streptomycin). After isolation from mixed glial cultures, the microglia cells were allowed to rest overnight prior to the treatments.

\section{Transfection}

For small interfering RNA (siRNA) transfection, primary microglia cells were transfected with FoxO1-specific siRNA (GenePharma, Shanghai, China) or negative control siRNA for $5 \mathrm{~h}$ via Lipofectamine 2000 (Invitrogen, Carlsbad, CA, USA) with Opti-MEM Reduced-Serum Medium (Gibco). Then, the medium was replaced with regular culture medium containing serum and the cells were cultured for $48 \mathrm{~h}$. Next, the microglia cells were exposed to LPS $(1 \mu \mathrm{g} / \mathrm{mL})$ with or without baicalin $(100 \mu \mathrm{M})$. A western blot analysis and immunofluorescence assay were used to evaluate the efficiency of siRNA.

\section{Immunocytochemistry}

BV2 cells and primary microglia cells were washed with cold PBS and fixed with $4 \%$ paraformaldehyde in PBS for $20 \mathrm{~min}$ and then permeabilized with $0.2 \%$ Triton X-100 for $15 \mathrm{~min}$. Non-specific binding was blocked by incubating the cells in PBS containing 10\% BSA. Cells were incubated with rabbit-anti-FoxO1 (1:100) or anti-TLR4 (1:100) overnight for $4{ }^{\circ} \mathrm{C}$. After three washings, the cells were incubated with goat anti-rabbit IgG H\&L (Alexa Fluor $488,1: 200$ ) at $37^{\circ} \mathrm{C}$ for $2 \mathrm{~h}$ and the nuclei were stained with DAPI for $10 \mathrm{~min}$. Fluorescent images were obtained using a confocal microscope.

\section{Enzyme-linked immunosorbent assay}

The levels of IL-1 $\beta$, IL-6, and TNF- $\alpha$ in the hippocampus of CUMS mice and the cell culture medium were measured with ELISA kits following the manufacturer's instructions. Data are shown in picograms per milligram protein (pg/mg prot).

\section{Cytoplasmic and nuclear protein extraction}

The cytoplasmic and nuclear proteins were extracted from fresh hippocampal tissues of CUMS mice and LPS-treated BV2 cells using nuclear and cytoplasmic protein extraction kits (product no: P0028, Beyotime Institute of Biotechnology, Shanghai, China). The extraction processes were strictly performed according to the manufacturer's instructions. The supernatants of the cytoplasmic and nuclear protein extractions were collected for subsequent experiments.

\section{Western blot analysis}

The proteins were extracted from the hippocampal tissues of CUMS mice, LPS-treated mice, and the treated cells, and the concentrations of the supernatants were quantified by the bicinchoninic acid assay according to the manufacturer's instructions (Beyotime, Nantong, China). The samples were separated by $10 \%$ SDS-PAGE and transferred onto polyvinylidene difluoride (PVDF) membranes and then blocked with 5\% skim milk in TBS-T (TBS containing $0.1 \%$ Tween-20). The membranes were incubated with the following primary antibodies: anti-TLR4 (1:1000), anti-p-PTEN (1:800), anti-PTEN (1:800), anti-p-PI3K (1:1000), anti-PI3K (1:1000), anti-p-AKT (1:1000), antiAKT (1:1000), anti-p-FoxO1 (1:800), anti-FoxO1 (1:1000), anti-p-AMPK (1:1000), anti-AMPK (1:1000), anti-GSK3 $\beta$ (1:1000), anti-p-GSK3 $\beta$ (1:800), anti- $\beta$-actin (1:1000), and anti-PCNA $(1: 1000)$ at $4{ }^{\circ} \mathrm{C}$ overnight. After incubation with anti-rabbit or anti-mouse secondary antibodies for $90 \mathrm{~min}$, the membranes were scanned by an imaging system (Bio-Rad, Hercules, CA, USA) and then quantified using ImageJ software (National Institutes of Health, USA).

\section{Statistical analysis}

Data are represented as the mean \pm SEM. The statistical analysis was conducted using the one-way ANOVA test followed by Tukey's test. A $p$ value $<0.05$ was considered significant. All of the data figures were obtained by GraphPad Prism 5.01 statistical software. 


\section{Results}

Baicalin reversed stress-induced depressive-like behavior by alleviating neuroinflammation

To investigate the effects of baicalin on CUMS-induced depressive-like behaviors of mice, we performed behavioral tests including SPT, OFT, TST, and FST as successively shown in Fig. 2a. In SPT and compared with the control mice, the consumption of sucrose was significantly reduced in CUMS-exposed mice $(p<0.01, F[5,66]=6.887)$. However, treatment with baicalin $(60 \mathrm{mg} / \mathrm{kg}, p<0.01)$ and Flu $(p<0.05)$ greatly reversed the abatement of the percentage of the sucrose preference, which reflected anhedonia (Fig. 2a, the first). Then, the locomotor activities were examined in the OFT. The CUMS mice showed a significant reduction in the number of crossings compared with the control group $(p<0.01, F[5,66]=6.122)$. Treatment with baicalin $(60 \mathrm{mg} / \mathrm{kg}, p<0.05)$ and Flu $(p<0.01)$ markedly improved the CUMS-induced lessening of locomotor activity (Fig. 2a, the second). No significant differences between the control group and the control+BA60 group indicated that baicalin had no significant effect on central excitability in normal mice. The desperate behaviors of mice were measured by recording the immobility time in the TST and FST. The CUMS mice observably increased the duration of immobility in TST $(p<0.01, F[5,66]=14.010$, Fig. $2 \mathrm{a}$, the third) and FST $(p<0.01, F[5,66]=4.734$, Fig. 2a, the fourth) compared to the control mice. As expected, the administration of baicalin $(30 \mathrm{mg} / \mathrm{kg}, p<0.05 ; 60 \mathrm{mg} / \mathrm{kg}, p<0.01)$ and Flu $(p<0.01)$ remarkably reduced the immobility time compared with the CUMS group. These results indicate that baicalin ameliorates CUMS-induced depressive-like behaviors.

Nissl staining was used to observe the morphological changes of the hippocampal neural cells in response to baicalin against CUMS-evoked neurotoxicity in mice. As shown in Fig. 2b first, neural cells in the hippocampal CA1 region were arrayed regularly and closely, and the Nissl bodies were clear in the control group. However, an observable ratio of neurons in the CUMS group was damaged with irregular and loose distribution as well as cytoplasm pyknosis and Nissl body disintegration. The numbers of Nissl-positive cells were observably reduced in the hippocampal CA1 region in the model mice compared with the control group ( $p<0.01, F[5,12]=7.397)$. In contrast, the severity of neuronal damage and the decrease of Nissl-positive cell numbers induced by CUMS were markedly ameliorated by baicalin $(60 \mathrm{mg} / \mathrm{kg}$, $p<0.01)$ and Flu $(p<0.05)$.

The above results show that baicalin pretreatment alleviated the CUMS-induced injury of the hippocampal neurons. We investigated the effects of baicalin on pro-inflammatory cytokines production in the hippocampus of CUMS mice. As shown in Fig. 2c, the levels of IL-1 $\beta(p<0.01, F[5,42]=6.410)$, IL-6 $(p<0.01, F[5,42]$
$=6.156)$, and TNF- $\alpha(p<0.01, F[5,42]=23.31)$ were evidently elevated in the CUMS group versus the control group, whereas these effects were significantly reversed by the treatment with Flu $(p<0.05)$ and baicalin $(60 \mathrm{mg} /$ $\mathrm{kg}, p<0.01)$. These data reveal that baicalin repressed the neuroinflammation in CUMS mice by downregulating hippocampal pro-inflammatory cytokines.

As described in Fig. 2b second, immunohistochemistry results showed that CUMS exposure also increased the expression of TLR4 in the hippocampal CA1 region versus the control group $(p<0.01, F[5,12]=10.15)$. Baicalin $(30$ $\mathrm{mg} / \mathrm{kg}, p<0.05 ; 60 \mathrm{mg} / \mathrm{kg}, p<0.01)$ and Flu $(p<0.05)$ administration observably inhibited the TLR4 expression compared with CUMS mice. Surprisingly, these results were mainly consistent with the anti-neuroinflammatory effect of baicalin, which indicates that TLR4 might play a role in this result.

\section{Role of TLR4 in the effect of baicalin in alleviating LPS- induced neuroinflammation}

To clarify the role of TLR4 in the anti-neuroinflammatory effect of baicalin on mice, we employed LPS (a main TLR4 ligand) to trigger inflammatory responses. TAK-242 is a specific inhibitor of TLR4 that works through disrupting the interactions of TLR4 with its adaptor molecules $[13,52]$. We used TAK-242 as a positive pharmacological tool to block the activity of TLR4.

As shown in Fig. 3a, the data of behavioral evaluations explicated that the activation of TLR4 by LPS evoked and exacerbated the depressive-like behaviors of mice. Compared to the control, LPS-injected mice showed a lower percentage of sucrose preference in SPT $(p<0.01, F[3,44]$ $=4.000)$, a lower locomotor activity in OPT $(p<0.005$, $F[3,44]=16.100)$, and a longer duration of immobility in TST and FST $(p<0.005, F[3,44]=7.628 ; p<0.005, F[3,44]$ $=10.540$, respectively). However, in comparison with the LPS group, the sucrose preference levels were higher in both LPS+TAK $(p<0.05)$ and the LPS+BA60 group. Similarly, mice in the LPS+TAK and LPS+BA60 groups were more active in OPT $(p<0.005 ; p<0.01$, respectively) versus mice in the LPS group. Moreover, shorter immobility times in TST were observed in the LPS+TAK $(p<0.005)$ and LPS + BA60 $(p<0.01)$ groups and shorter immobility durations in FST were expressed in the LPS+TAK $(p<0.01)$ and LPS + BA60 $(p<0.005)$ groups when compared to the LPS group.

As presented in Fig. 3c, the activation of TLR4 by LPS observably promoted the production of pro-inflammatory cytokines in the hippocampus of LPS-treated mice. In parallel with the results of behavioral evaluations, the LPS injection greatly enhanced the levels of IL-1 $\beta(p<0.05$, $F[3,28]=4.292), \quad$ IL-6 $\quad(p<0.01, \quad F[3,28]=8.498)$, and TNF- $\alpha(p<0.01, F[3,28]=6.870)$ in the hippocampus of the LPS group of mice versus the control group. The 


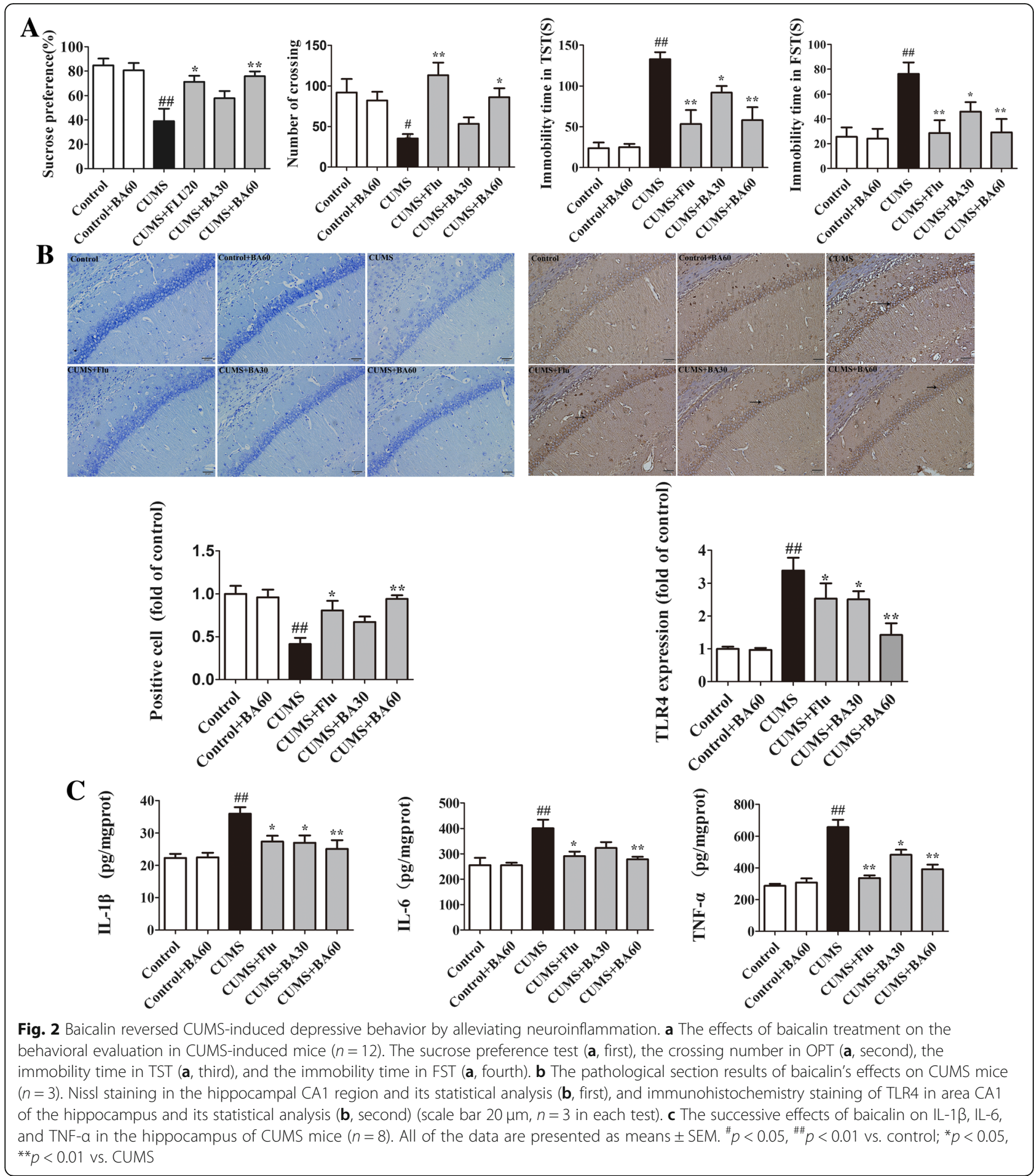

TAK-242 lessened the expression of IL-1 $\beta$, IL- 6 , and TNF- $\alpha$ by blocking the biological effect of TLR4. In addition, the baicalin treatment represented the same efficacy with TAK-242 administration in LPS-induced neuroinflammation.

Data from Nissl staining are shown in Fig. 3b first. The Nissl bodies became small and indistinct, and the number of Nissl-positive cells significantly lessened in the hippocampal CA1 region of LPS-treated mice $(p<$ $0.01, F[3,8]=17.490)$ versus the control group. In addition, the treatment with TAK-242 $(p<0.05)$ and baicalin $(p<0.05)$ augmented the number of Nissl-positive cells, which indicated that TAK-242 and baicalin reduced the LPS-induced injury of hippocampal neurons. 

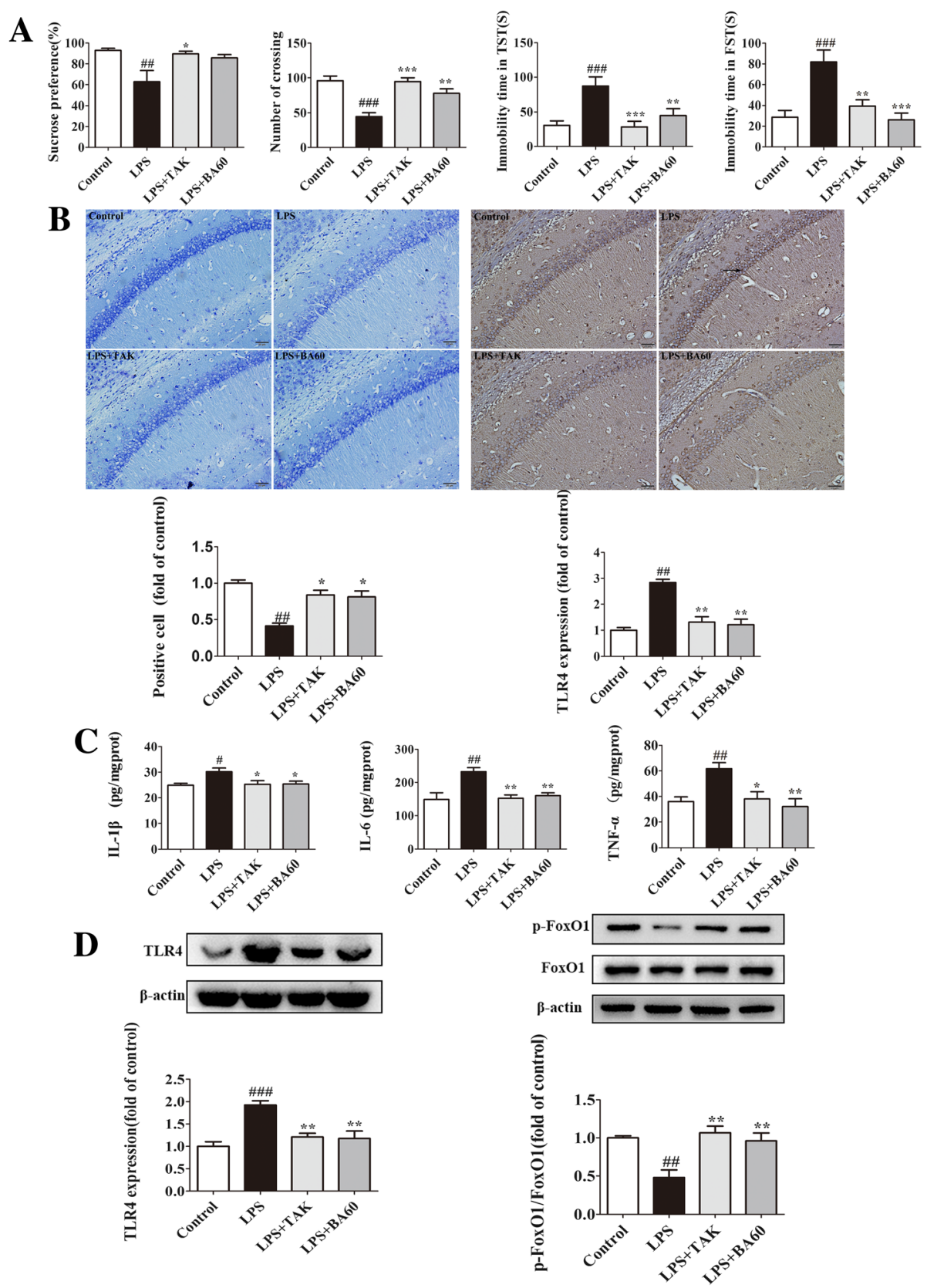

Fig. 3 The role of TLR4 in alleviating neuroinflammation of baicalin. Prophylactic treatment of TAK-242 or baicalin inhibited LPS-induced neuroinflammation as well as depressive-like behaviors. a Pretreatment of TAK-242 or baicalin ameliorated LPS-induced depressive behaviors, which were successively measured by SPT, OPT, TST, and SFT ( $n=12$ in each test). $\mathbf{b}$ Representative pathological images of Nissl staining and immunohistochemistry staining of TLR4 in the hippocampal CA1 subregion ( $n=3$ in each test; scale bar $20 \mu \mathrm{m}$ ). c The levels of IL-1 $\beta$, IL-6, and TNF-a in the hippocampus were measured by ELISA kits $(n=8)$. $\mathbf{d}$ The levels of TLR4 and p-FoxO1 in the hippocampus were measured by western blotting and were quantified and normalized with their respective $\beta$-actin or total FoxO1 levels $(n=4)$. Data were normalized to the control and presented as means \pm SEM. ${ }^{\#} p<0.05,{ }^{\# \#} p<0.01,{ }^{\# \# \#} p<0.005$ vs. control; ${ }^{*} p<0.05,{ }^{* *} p<0.01,{ }^{* * *} p<0.005$ vs. LPS. TAK, TAK-242

Immunohistochemistry staining of TLR4 in area CA1 of the hippocampus showed that LPS stimulation elicited the activation of TLR4 and upregulated its expression as exhibited in Fig. 3b second $(p<0.01, F[3,8]=24.65)$. TAK-242 inhibited LPS-induced TLR4 expression by blocking the TLR4 signaling pathway $(p<0.01)$. Similar to TAK-242, baicalin also downregulated the expression of TLR4 induced by LPS $(p<0.01)$.

Previous research showed that FoxO1 binds to multiple enhancer-like elements within the TLR4 gene and regulates the transcription of TLR4 [23]. We further investigated the interaction between TLR4 and FoxO1 in 
LPS-treated mice. As shown in Fig. 3d, LPS injection significantly enhanced the expression of TLR4 $(p<0.005$, $F[3,12]=12.28)$ and the activity of FoxO1 by downregulating its phosphorylation $(p<0.01)$ in the hippocampus. TAK-242 blocked the LPS-induced elevation of TLR4 expression $(p<0.01)$ and the activity of FoxO1 through upregulation of the level of $\mathrm{p}$-FoxO1 $(p<0.01)$. Furthermore, baicalin also decreased the expression of TLR4 $(p<0.01)$ and the activity of FoxO1. These data indicate that the blocking of TLR4 was accompanied by a higher level of p-FoxO1 and a lower transcriptional activity of FoxO1.

The activation of TLR4 could trigger neuroinflammation and evoke depression-like behaviors. Inhibition of the TLR4 signaling pathway by TAK-242 alleviated neuroinflammation and depressive behaviors induced by LPS. Baicalin ameliorated LPS-induced neuroinflammation and reversed LPS-evoked depressive-like behaviors by downregulating the expression of TLR4. Furthermore, FoxO1 might be involved in the effect of baicalin on TLR4 expression. This evidence indicates that TLR4 has a vital role in the alleviation of neuroinflammation and depressive-like behaviors by baicalin.

\section{Baicalin reduced CUMS-induced TLR4 expression and inflammatory responses by activating the PI3K/AKT pathway in mice}

$\mathrm{PI} 3 \mathrm{~K} / \mathrm{AKT}$ is the primary signaling transduction pathway responsible for the synthesis and production of pro-inflammatory mediators and can even regulate the expression of TLR4 [54-56]. We treated LY294002 (a PI3K/Akt pathway inhibitor) $1 \mathrm{~h}$ before the administration of baicalin in CUMS mice to investigate whether baicalin downregulated the expression of TLR4 in CUMS mice via the PI3K/AKT pathway.

The efficacy of baicalin treatment on CUMS mice was dramatically reversed by the prophylactic administration of LY294002, which is explicated in the behavioral tests in Fig. 4a. The baicalin administration greatly attenuated depressive-like behaviors observed in CUMS-induced mice. However, in comparison with the CUMS+BA60 group, the CUMS+LY+BA60 group mice showed a lower percentages of sucrose preference in SPT $(p<0.05, F[3,44]=9.594)$, a lower locomotor activity in OPT $(p<0.05, F[3,44]=8.022)$, and a longer duration of immobility in TST $(p<0.01$, $F[3,44]=18.00)$ and FST $(p<0.05, F[3,44]=9.860)$. These data indicate that the antidepression effect of baicalin in CUMS mice was blocked by LY294002.

In addition, baicalin inhibited the CUMS-induced production of IL-1 $\beta$, IL- 6 , and TNF- $\alpha$ in hippocampal tissues, but LY294002 $(p<0.05$ in IL-6 and TNF- $\alpha)$ abolished the anti-neuroinflammatory effects of baicalin (Fig. 4c).

As the Nissl staining results showed in Fig. 4b first, the change of the neuronal morphology and the reduction of Nissl-positive cells in the hippocampal CA1 subregion of CUMS mice were ameliorated by the baicalin treatment $(p<0.01, F[3,8]=24.53)$. The protective effect of baicalin could be abrogated by the pretreatment of LY294002 $(p<0.01)$. Meanwhile, the increase of TLR4 induced by CUMS exposure in the CA1 region of the hippocampus was remarkably lessened by baicalin, but the levels of TLR4 were markedly increased after LY294002 treatment $(p<0.05, F[3,8]=13.89)$ as shown by the immunohistochemistry staining of TLR4 in Fig. 4b second .

The western blot results showed that CUMS exposure robustly repressed the phosphorylation of PI3K $(p<$ $0.005, F[3,12]=23.11)$ and AKT $(p<0.01, \quad F[3,12]=$ $28.53)$ and exacerbated the expression of TLR4 $(p<0.01$, $F[3,12]=14.33)$ of the CUMS group mice versus the control group as described in Fig. 4c. The administration of baicalin $(60 \mathrm{mg} / \mathrm{kg})$ greatly elevated the expression of p-PI3K $(p<0.01)$ and p-AKT $(p<0.01)$ and significantly restrained the expression of TLR4 $(p<0.05)$ in CUMS mice. Nevertheless, LY294002 abolished the effects of baicalin by blocking the activation of PI3K/AKT and was responsible for the increase of TLR4 expression $(p<$ 0.05) in the CUMS+LY+BA60 group. Collectively, these findings provide direct evidence that baicalin repressed the incremental expression of TLR4 and inflammatory responses induced by CUMS via activation of the PI3K/ AKT pathway.

\section{Baicalin regulated CUMS-induced TLR4 expression by activating the $\mathrm{PI} 3 \mathrm{~K} / \mathrm{AKT} / \mathrm{FoxO} 1$ pathway in vivo}

To further investigate the potential mechanism of baicalin on CUMS-evoked depression, the protein levels of PI3K/AKT-related signaling in the hippocampus of CUMS mice were examined by western blot. As shown in Fig. 5a and in comparison with the control group, CUMS robustly increased the expression of TLR4 $(p<$ $0.01, F[5,18]=6.188)$ and then substantially decreased the expression of p-PTEN $(p<0.01, F[5,18]=7.265)$, p-PI3K $(p<0.01, F[5,18]=6.778), \quad$-AKT $(p<0.01$, $F[5,18]=14.530)$, and $\mathrm{p}-\mathrm{FoxO} 1 \quad(p<0.01, \quad F[5,18]=$ 16.240). Treatment with Flu $(p<0.05)$ and baicalin $(60$ $\mathrm{mg} / \mathrm{kg}, p<0.01)$ significantly reversed CUMS-elicited alterations in the expression of TLR4, p-PTEN, p-PI3K, and $\mathrm{p}$-AKT compared with the CUMS group. As for p-FoxO1, although Flu did not completely alter the downtrend of p-FoxO1 expression, it still improved the situation induced by CUMS, and baicalin $(60 \mathrm{mg} / \mathrm{kg}) \mathrm{ab}-$ solutely changed the downtrend of $\mathrm{p}$-FoxO1 expression induced by CUMS $(p<0.01)$. However, the Flu and baicalin $(60 \mathrm{mg} / \mathrm{kg})$ treatments had no significant effects on the levels of $\mathrm{t}$-FoxO1 in CUMS mice.

FoxO1 is a transcriptional factor binding to multiple enhancer-like elements within the TLR4 gene [23], and its nuclear localization is modulated by phosphorylation. 
A
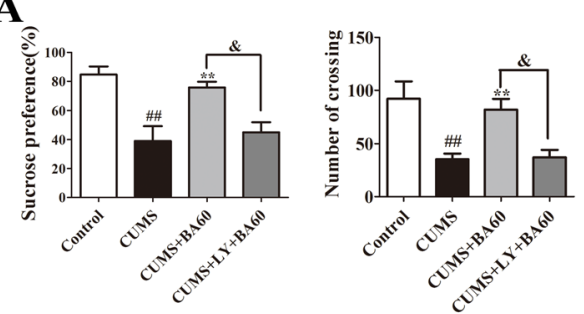

B
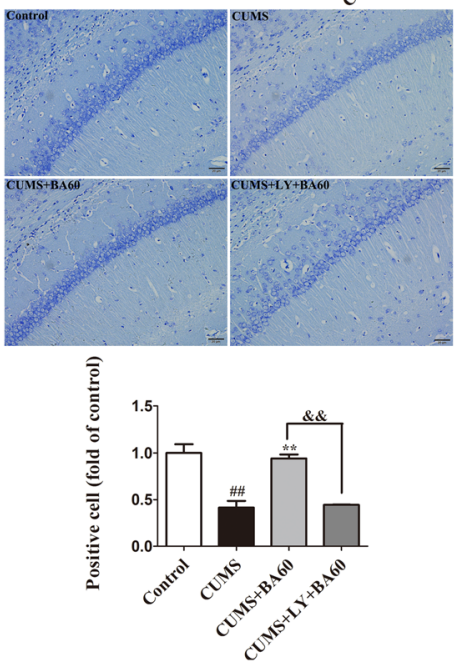

C

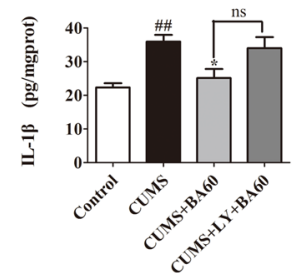

D

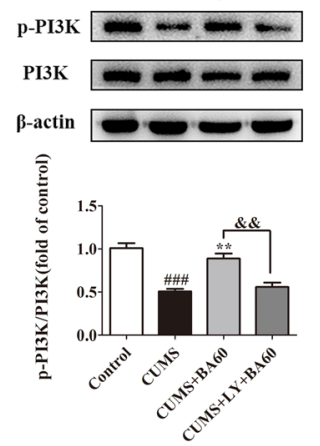

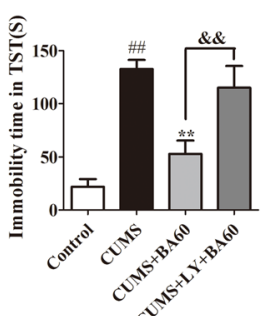
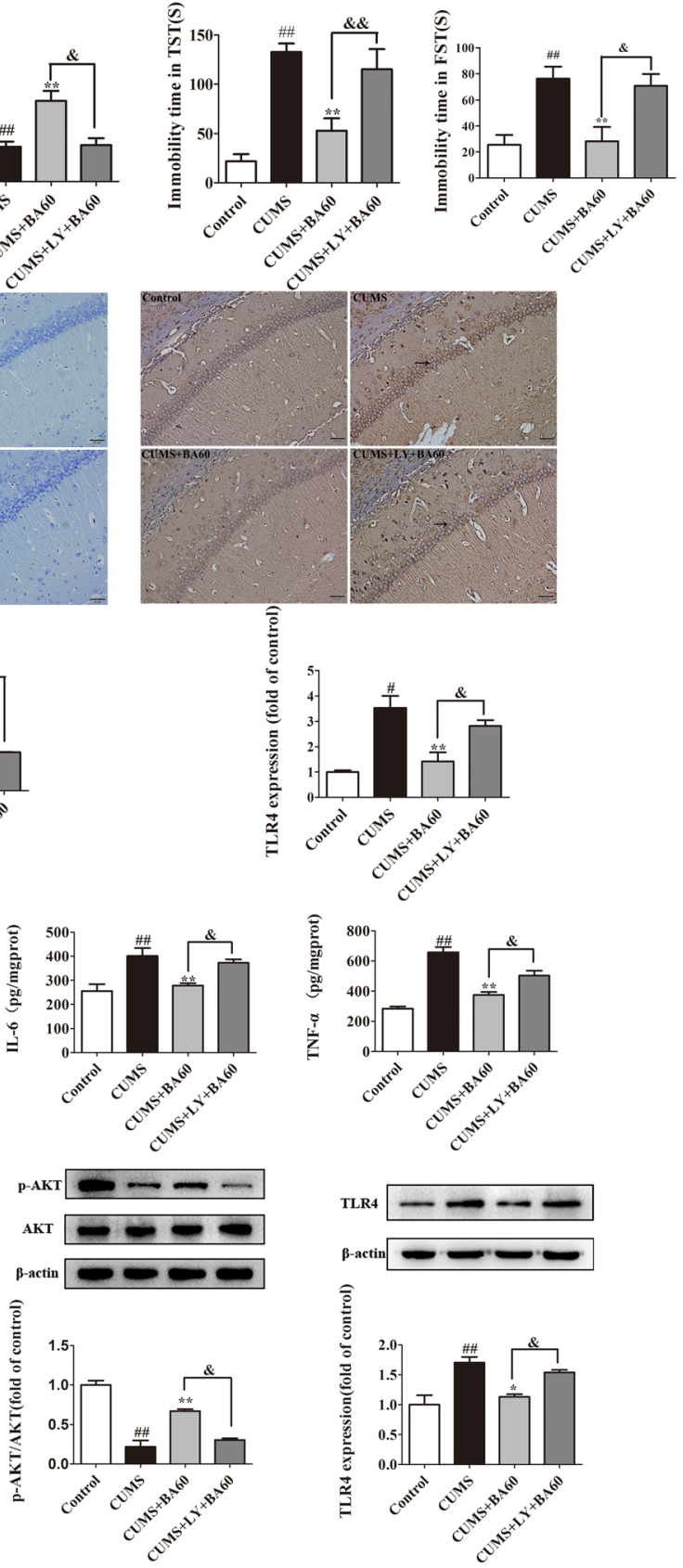

Fig. 4 Baicalin reduced the CUMS-induced TLR4 expression and inflammatory response by activating PI3K/AKT. LY294002 was employed to abolish the effect of baicalin on PI3KJAKT in CUMS mice, and the mice were allocated into four groups: the control, CUMS, CUMS+BA60 and CUMS+LY+BA60. a The behavioral evaluation results including SPT, OPT, TST, and OPT $(n=12)$. b The Nissl staining results in the hippocampal CA1 region and the statistical analysis (b, first) and TLR4 immunohistochemistry staining in the hippocampal CA1 subregion and its statistical analysis (b, second) (scale bar $20 \mu \mathrm{m} ; n=3$ in each experiment). $\mathbf{c}$ The levels of IL-1 $\beta$, IL-6, and TNF-a in the hippocampus were determined by ELISA kits $(n=8)$. $\mathbf{d}$ The western blotting results of p-PI3K, p-AKT, and TLR4 were quantified $(n=4)$. Data were normalized to the control and presented as means \pm SEM. ${ }^{\#} p<0.05,{ }^{\# \#} p<0.01,{ }^{\# \# \#} p<0.005$ vs. control; ${ }^{*} p<0.05,{ }^{*} p<0.01$ vs. CUMS; ${ }^{*} p<0.05,{ }^{\& \&} p<0.01$ vs. CUMS+BA60. LY, LY294002; BA60, baicalin treatment with $60 \mathrm{mg} / \mathrm{kg}$

Thus, the subsequent experiments were designed to investigate the nuclear translocation of FoxO1 by detecting the levels of p-FoxO1 and FoxO1 in the cytoplasmic and nuclear fractions, respectively, of hippocampal tissues of
CUMS mice. As exhibited in Fig. 5b, the levels of $\mathrm{p}$-FoxO1 in the cytoplasmic protein extractions were strongly weakened after CUMS exposure in contrast to the control $(p<0.01, F[5,18]=12.25)$, and treatment with 


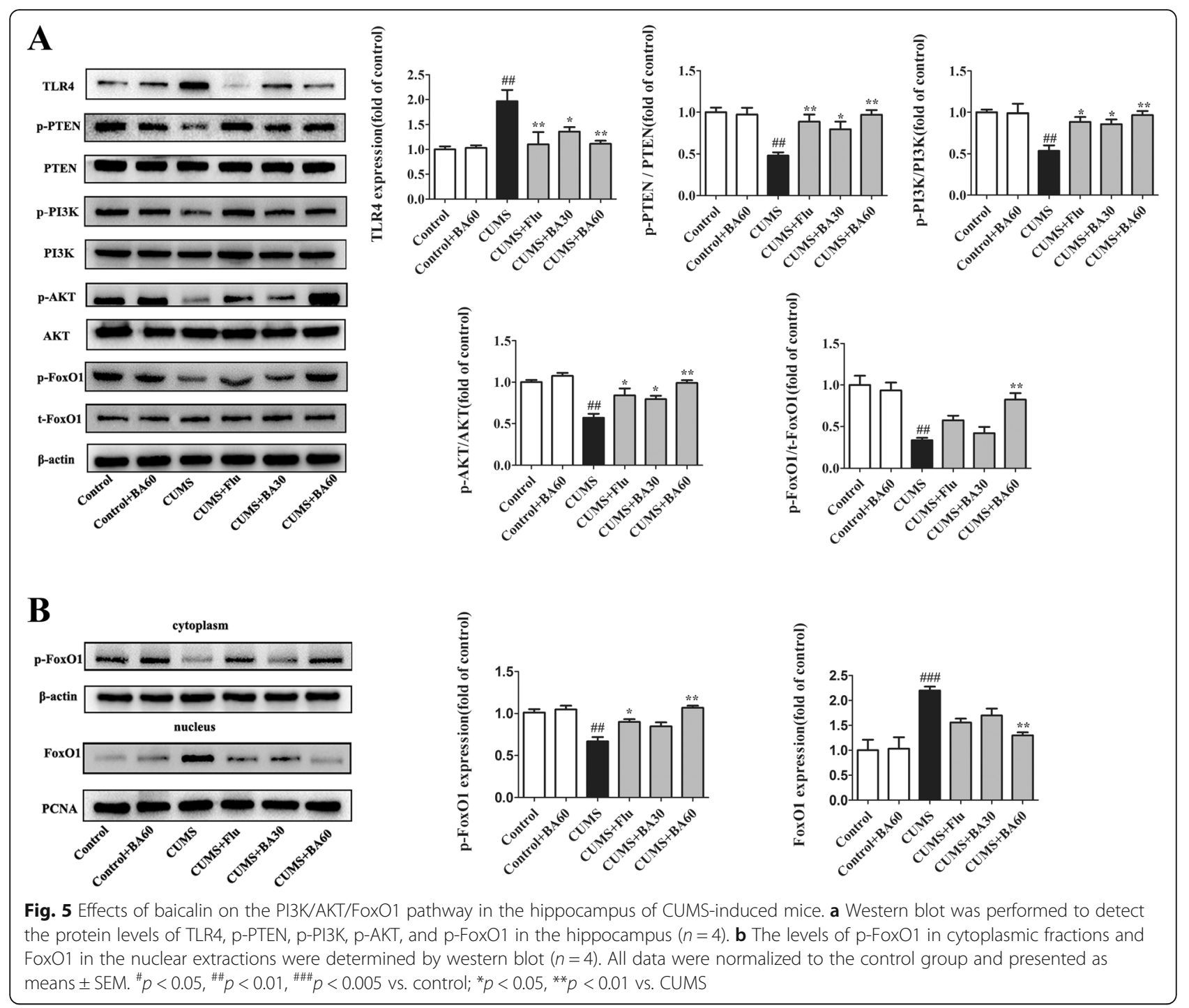

Flu $(p<0.05)$ or baicalin $(60 \mathrm{mg} / \mathrm{kg}, p<0.01)$ increased the levels of p-FoxO1 in the cytoplasm compared with CUMS. Correspondingly, the levels of FoxO1 in the nuclear fractions extracted from the hippocampus of the CUMS group mice were significantly higher than those of the control group mice $(p<0.005, F[5,18]=10.22)$. The CUMS-induced uptrend of the FoxO1 level in the nuclear extractions was dramatically reversed by the administration of baicalin $(60 \mathrm{mg} / \mathrm{kg}, p<0.01)$ and could be altered (to a certain extent) by the application of Flu. These data indicate that baicalin could indirectly promote the phosphorylation of FoxO1 and then promote its nuclear exclusion and subsequent sequestration into the cytoplasm in CUMS-induced mice, which was responsible for inhibiting FoxO1 transcription activity and then repressing the expression of TLR4.

To explore the role of PI3K/AKT signaling in the repression of TLR4 expression by baicalin, we employed
LY294002 to block the PI3K/AKT pathway. From the western blot data in Fig. 6a, the inhibition of PI3K by LY294002 efficiently abrogated the effects of baicalin on p-PI3K $(p<0.01, \quad F[3,12]=22.16), \quad$ p-AKT $\quad(p<0.01$, $F[3,12]=39.36)$, p-FoxO1 $(p<0.05, F[3,12]=10.50)$, and TLR4 $(p<0.05, F[3,12]=17.43)$ expression in the hippocampus of the CUMS group mice. In addition, the inhibition of PI3K/AKT activation was responsible for the dephosphorylation of FoxO1 and increased its nuclear accumulation and activity as exhibited in Fig. 6b. Altogether, these results suggest that baicalin could inhibit FoxO1 transactivation via activation of PI3K/AKT signaling and then inhibit the expression of TLR4 in CUMS mice.

\section{Baicalin regulated LPS-induced TLR4 expression by} activating the PI3K/AKT/FoxO1 pathway in BV2 cells Microglial cells are the main source of pro-inflammatory factors in the brain after stress. Therefore, it is worthwhile 

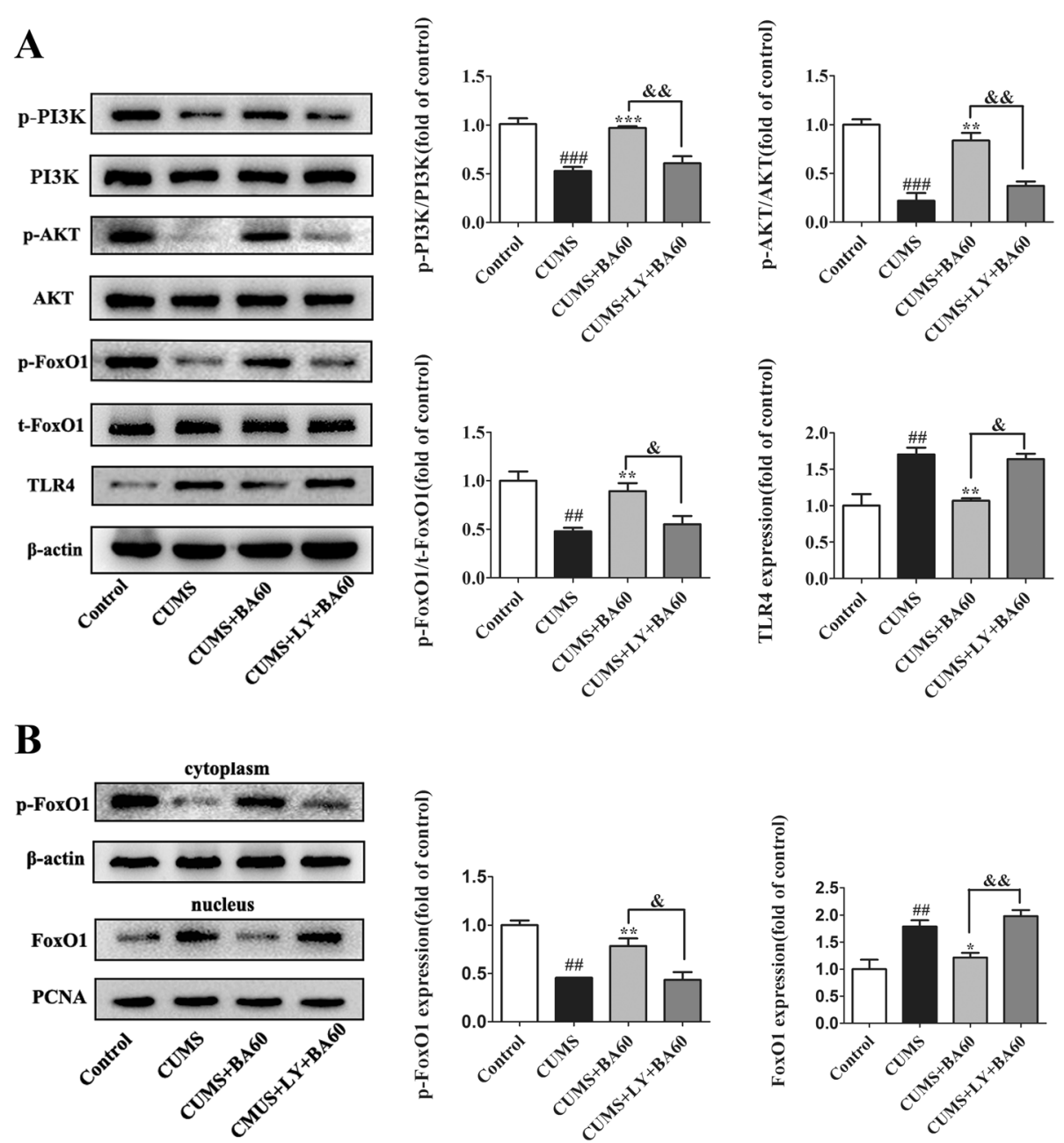

Fig. 6 Baicalin inhibited FoxO1 transactivation and TLR4 expression in the hippocampus of CUMS mice in a manner dependent on PI3K/AKT. a Western blot was performed to determine the levels of p-PI3K, p-AKT, p-FoxO1, and TLR4 in the hippocampus of CUMS mice after treatment with baicalin and LY294002 $(n=4)$. b Representative protein bands of p-FoxO1 in the cytoplasmic fractions and FoxO1 in the nuclear extractions. Data were normalized to the control group and presented as means \pm SEM. ${ }^{\#} p<0.05$, ${ }^{\# \#} p<0.01,{ }^{\# \# \#} p<0.005$ vs. control; ${ }^{*} p<0.05$, ${ }^{* *} p<0.01$ vs. CUMS; ${ }^{\&} p<0.05,{ }^{\&} \& p 0.01$ vs. CUMS+BA60

to perform extracorporeal validation in BV2 microglial cells. The LPS, specifically combined with TLR4, was employed to trigger inflammatory responses in BV2 cells. As described in Fig. 7c, the BV2 cells were incubated with LPS $(1 \mu \mathrm{g} / \mathrm{mL})$ for $24 \mathrm{~h}$, and the ensuing production of IL-1 $\beta(p<0.01, F[3,28]=51.86)$, IL-6 $(p<0.01, F[3,28]=$ $48.93)$, and TNF- $\alpha(p<0.01, F[3,28]=30.78)$ robustly increased in the LPS group versus the blank group. Pretreatment with baicalin $(100 \mu \mathrm{M})$ could efficiently change their uptrend; however, LY294002 $(10 \mu \mathrm{M})$ significantly abolished the effects of baicalin.

As the western blot results show in Fig. 7a, the expressions of p-PI3K $(p<0.01, F[3,12]=26.46)$, p-AKT $(p<0.01$, $F[3,12]=37.41)$, and p-FoxO1 $(p<0.01, \quad F[3,12]=11.18)$ were strongly weakened and the level of TLR4 $(p<0.01$, $F[3,12]=10.09)$ was robustly higher after LPS administration in contrast to the blank group. The application of baicalin $(p<0.05)$ greatly reversed these alterations in the LPS-treated BV2 cells, and the protective effects of baicalin could be abolished by the incubation with LY294002.

Figure $7 \mathrm{~b}$ demonstrates that LPS raised the activity of FoxO1 by increasing the dephosphorylation of FoxO1 and its nuclear accumulation. When the BV2 cells were treated with baicalin, the phosphorylation of FoxO1 was enhanced and its nuclear exclusion was promoted. However, the augmented effect of baicalin on FoxO1 phosphorylation was also weakened by LY294002. Likewise, Fig. 7d also showed that baicalin inhibited FoxO1 transcriptional activity in LPS-incubated BV2 cells by promoting FoxO1 nuclear exclusion and then substantially decreased the LPS-induced TLR4 expression (Fig. 7e). The application of LY294002 abolished the effect of 


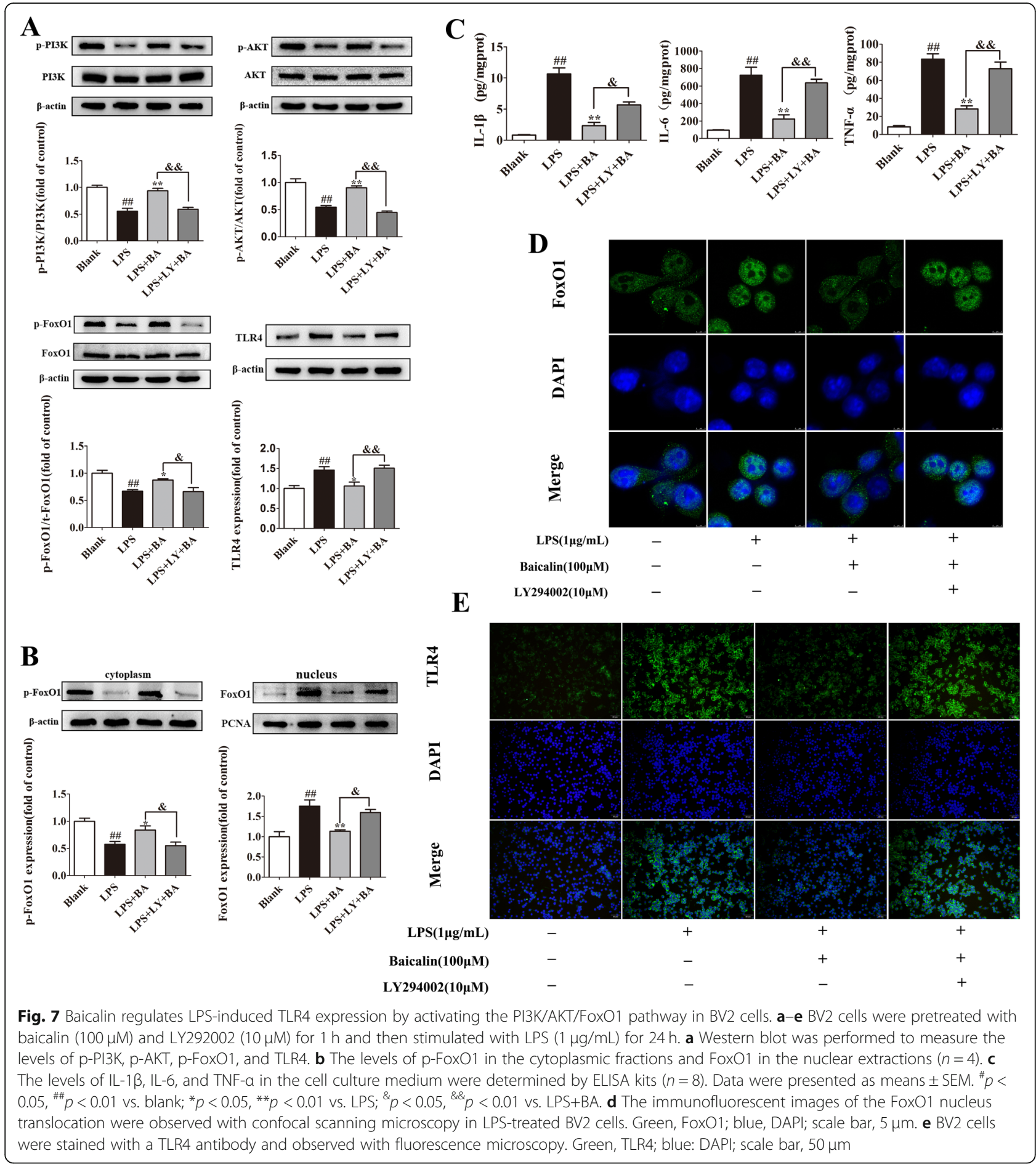

baicalin on FoxO1 nuclear exclusion (Fig. 7d) and then substantially increased the expression of TLR4 (Fig. 7e). Collectively, these results demonstrate that baicalin downregulated the LPS-induced TLR4 expression by activating the PI3K/AKT/FoxO1 pathway in BV2 cells.
Baicalin regulated LPS-induced TLR4 expression by activating the $\mathrm{PI3K} / \mathrm{AKT} / \mathrm{FoxO} 1$ pathway in primary microglia cells

To confirm the data obtained from BV2 microglial cells, we further proved the above findings in primary mouse microglia cells. As the western blot results described in 
Fig. 8a, in response to LPS stimulation, we observed a decrease in the protein levels of p-PI3K $(p<0.01, F[3,8]$ $=12.81)$, $\mathrm{p}$-AKT $(p<0.01, F[3,8]=14.25)$, and $\mathrm{p}$-FoxO1 $(p<0.05, F[3,8]=8.319)$ and an increase of TLR4 $(p<0.01, \quad F[3,8]=11.67)$ in LPS-treated primary microglia cells. Pretreatment with baicalin $(100 \mu \mathrm{M})$ resulted in a significant upregulation of p-PI3K $(p<0.05)$, p-AKT $(p<0.01)$, and p-FoxO1 $(p<0.05)$. Since the phosphorylation of FoxO1 was enhanced, the activity of FoxO1 was inhibited, which resulted in its nuclear exclusion in primary microglia cells as shown in Fig. 8b. In addition, the regulating transcriptional effects of FoxO1 on TLR4 $(p<0.05)$ gene expression were also inhibited by baicalin as exhibited in Fig. 8a and c. However, application of LY294002 $(10 \mu \mathrm{M})$ abolished the effect of baicalin on FoxO1 nuclear exclusion (Fig. 8b) and on downregulation expression of TLR4 (Fig. 8a, c). These results reconfirmed that the anti-neuroinflammatory effect of baicalin was mediated by the PI3K/AKT/FoxO1 pathway. Surprisingly, these results were mainly consistent with the experiments described above and again indicate that baicalin regulated the incremental expression of TLR4 and inflammatory responses induced by stress via activation of the PI3K/AKT/ FoxO1 pathway.

\section{Fox01 played a vital role in baicalin regulation of the TLR4 signaling}

The transcriptional activity of FoxO1 was inhibited via phosphorylation by $\mathrm{PI} 3 \mathrm{~K} / \mathrm{AKT}$, which leads to the nuclear translocation and inhibition of target gene expression. To verify whether FoxO1 was involved in baicalin regulation of the TLR4 signaling, we knocked down the expression of FoxO1 using RNA interference (RNAi) based on the primary microglia cells. The primary microglia cells were transfected with FoxO1-siRNA for $5 \mathrm{~h}$, and after resting overnight, the cells were treated with baicalin and LPS for $24 \mathrm{~h}$. The FoxO1 was successfully inhibited by FoxO1-siRNA compared with the control-siRNA $(p<0.01, \quad F[2,9]=17.31)$ as well as non-treated cells $(p<0.01)$ (Fig. 9a, b). As described in Fig. 9c, the transfection of FoxO1-siRNA significantly strengthened the effects of baicalin on LPS-induced TLR4 expression $(\& \& p<0.01, F[4,15]=59.42)$, while it had no significant effects on the expression of p-PI3K and $\mathrm{p}-\mathrm{AKT}$. These results indicate that FoxO1 is a crucial downstream target of AKT. However, the increases of the TLR4 levels induced by LPS were abrogated by FoxO1-siRNA $(\$ \$ p<0.005)$. Likewise, the production of IL-1 $\beta\left({ }^{\$ \$} p<0.005, F[4,25]=59.23\right)$, IL-6 $\left({ }^{\$ \$} p<0.005\right.$, $F[4,25]=104.9)$, and TNF- $\alpha \quad(\$ \$ p<0.005, \quad F[4,25]=$ 133.0) induced by LPS in primary microglia cells was also abolished by FoxO1-siRNA as shown in Fig. 10d. Meanwhile, the FoxO1-siRNA significantly strengthened the inhibitory effects of baicalin on the LPS-induced production of IL-6 $\left({ }^{\&} p<0.05\right)$ and TNF- $\alpha\left({ }^{\& \&} p<0.01\right)$ in primary microglia cells. Thus, it could be inferred that FoxO1 played a vital role in the inhibitory effect of baicalin on TLR4 expression and TLR4-mediating inflammation signaling triggered by LPS.

\section{Discussion}

The anti-inflammatory effects of baicalin in peripheral inflammatory diseases have been broadly investigated and might be linked to the inhibition of TLR4 and its downstream signaling [25-28]. Current research studies indicate that neuroinflammation plays a vital role in the pathogenesis of depressive disorders $[9,57]$. In this study, we found that the chronic application of baicalin exhibited antidepressant effects by ameliorating neuroinflammation in the CUMS model of depressive mice, and the underlying mechanisms were associated with the inhibition of TLR4 expression via the PI3K/AKT/FoxO1 pathway.

Considering that stress induced the production of pro-inflammatory cytokines, which might evoke a depressive-like state in animal model systems [3], it is reasonable to presume that stress-induced increases of inflammation might play a role in depression. In our study, the levels of IL-1 $\beta$, IL- 6 , and TNF- $\alpha$ were substantially increased in the hippocampus of the depression mice model induced by CUMS. However, the administration of baicalin $(60 \mathrm{mg} / \mathrm{kg})$ apparently ameliorated CUMS-induced depressive-like symptoms and greatly decreased pro-inflammatory cytokines in the hippocampus while concurrently downregulating TLR4 expression. These data allude to the fact that baicalin could inhibit neuroinflammation and TLR4 might participate in this process.

TLR4 regulates the adrenal responses to stress and inflammatory stimuli as well as brain responses to stress $[15,16]$. Previous studies demonstrated that stress exposure upregulated the TLR4 pathway, and this suggests a vital role of TLR4 in the production of neuroinflammation in the brain [58]. In this study, we substantiated the fact that TLR4 indeed participated in the anti-neuroinflammatory and antidepressive effects of baicalin as follows: (i) activation of TLR4 by LPS-triggered robust elevation of IL- $1 \beta$, IL- 6 , and TNF- $\alpha$ in the hippocampus and injury of hippocampal neurons as well as depressive symptoms in mice; (ii) blocking TLR4 by TAK-242 reversed LPS-induced neuroinflammation and depressivelike behaviors; and (iii) baicalin efficiently inhibited neuroinflammation and the expression of TLR4 induced by the specific activation of TLR4 with LPS, and baicalin also ameliorated behavioral changes induced by LPS with a similar efficacy of TAK-242.

Previous studies reported that the activation of TLR4 by LPS or HMGB1 resulted in the activation of PTEN through dephosphorylation $[49,59,60]$. PTEN is a 

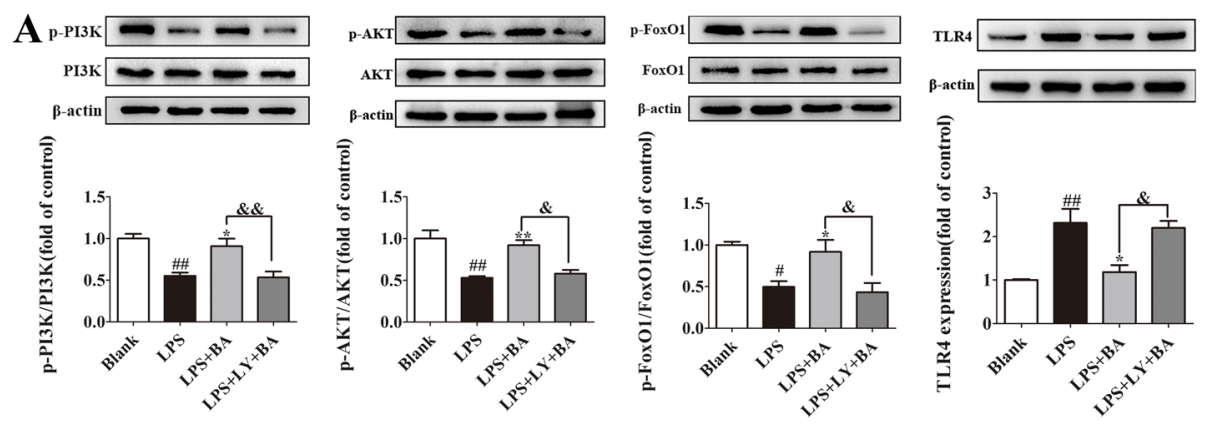

B
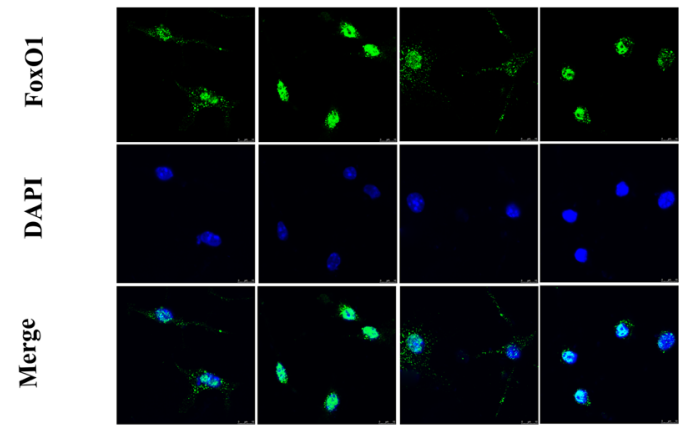

$\operatorname{LPS}(1 \mu \mathrm{g} / \mathrm{mL})$

Baicalin $(100 \mu \mathrm{M})$

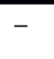

$-$

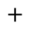

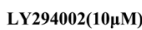

$-$

$-$

$-$

$+$

C

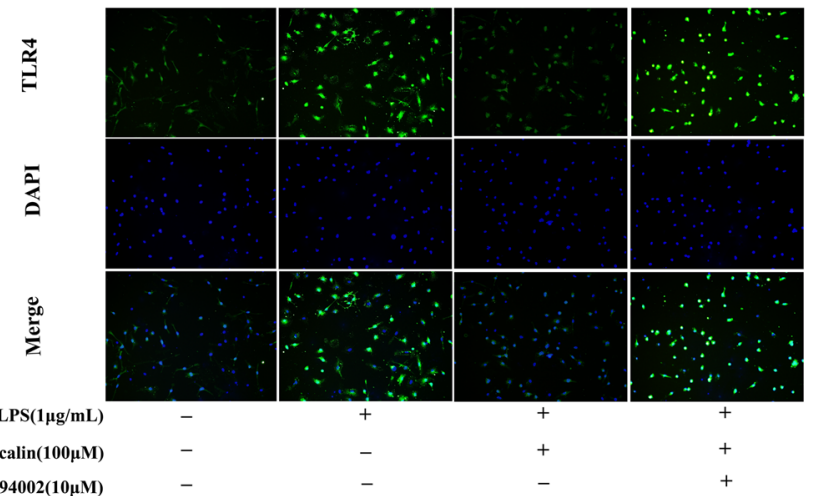

Fig. 8 Baicalin regulates LPS-induced TLR4 expression by activating the PI3KVAKT/FoxO1 pathway in primary microglia cells. Primary microglia cells were pretreated with baicalin and LY 294002 for $1 \mathrm{~h}$ and then stimulated with LPS for $24 \mathrm{~h}$. a Western blot was performed to measure the levels of p-PI3K, p-AKT, p-FoxO1, and TLR4. Data were normalized to the blank group and presented as means \pm SEM. " $p<0.05$, \#\# $p<0.01$ vs. blank; ${ }^{*} p<0.05,{ }^{* *} p<0.01$ vs. LPS; ${ }^{\&} p<0.05,{ }^{\&} p<0.01$ vs. LPS+BA. b The immunofluorescent images of FoxO1 nucleus translocation were observed by confocal scanning microscopy in LPS-treated primary microglia cells. Green, FoxO1; blue: DAPl; scale bar 10 um. c Primary microglia cells were stained with a TLR4 antibody and observed with fluorescence microscopy. Green, TLR4; blue, DAPI; scale bar 50 m

tumor suppressor with dual phosphatase activity and expression in the neurons in the human, mouse, and rat brain, and has a functional role in the CNS development by regulating cell senescence, apoptosis, inflammation, and other pathophysiological processes [61]. PTEN is known to be a major negative regulator of PI3K/AKT by dephosphorylating PIP3 to PIP2, which results in antagonizing PI3K and then inactivating AKT [62, 63]. Accordingly, our results demonstrate that the CUMS stressors activated TLR4, which led to the increase of TLR4 and the decrease of p-PTEN (inactive form) expression and promoted the activation of PTEN and then inhibition of the phosphorylation of AKT (Fig. 5). However, treatment with baicalin decreased the expression of TLR4 and elevated the expression of p-PI3K and p-AKT, which could be related to the activation of the PI3K/ AKT signaling pathway. Interestingly, previous studies indicated that the phosphorylation of PI3K/AKT, as a downstream target of TLR4, was dependent on LPS-stimulated TLR4 activation in a time-dependent manner and reached a maximum level $1 \mathrm{~h}$ after LPS stimulation [55, 64]. This might be a transient self-limiting feedback mechanism. However, in CUMS mice, accumulating PTEN might play a predominant 
A
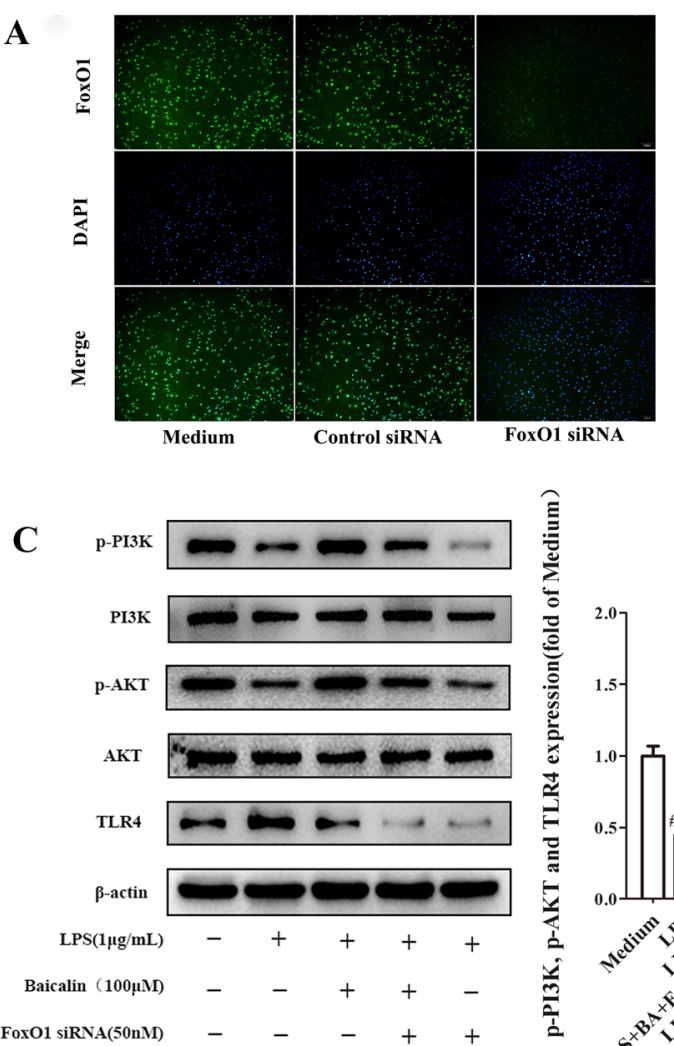
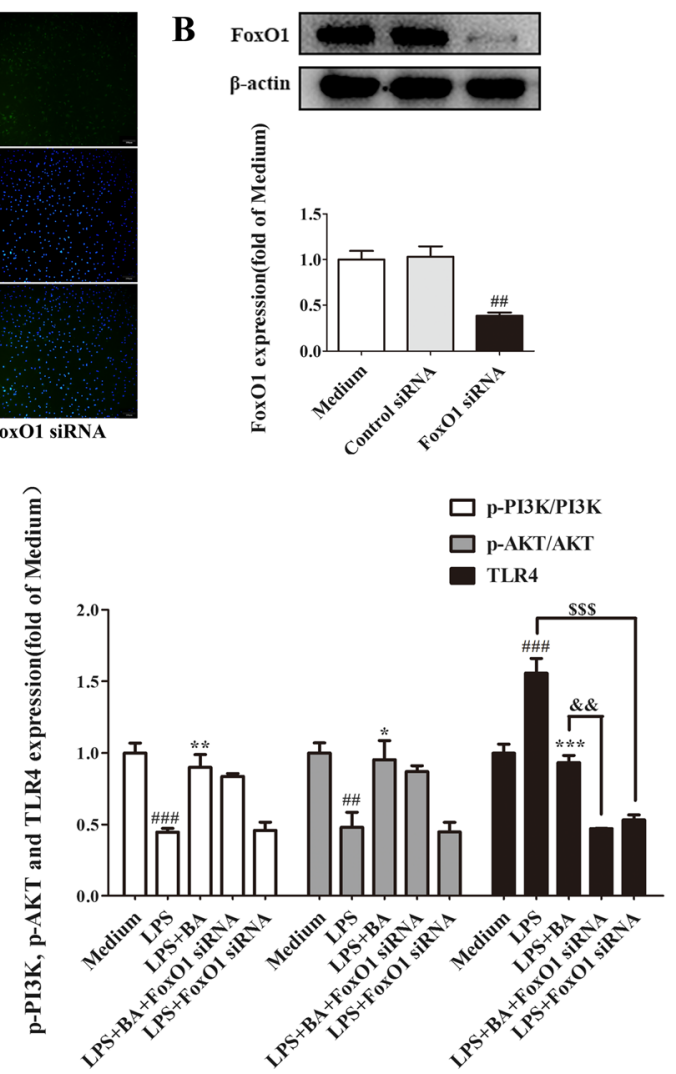

D
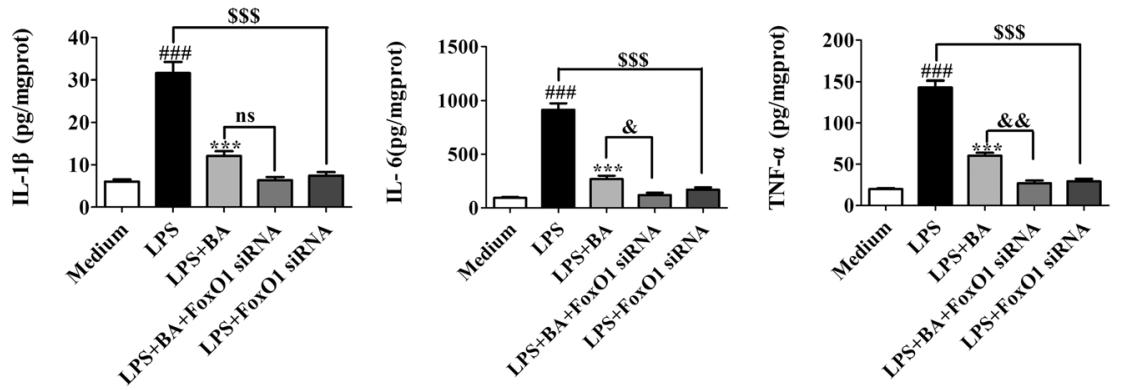

Fig. 9 The knockdown of FoxO1 enhanced the inhibitory effect of baicalin on LPS-induced TLR4 expression and inflammation. a-d The knockdown of FoxO1 expression by FoxO1 siRNA. a The cells were stained with FoxO1 (green) antibody and observed using fluorescence microscopy (scale bar $100 \mu \mathrm{m}$ ). $\mathbf{b}$ Western blotting analysis of the expression of FoxO1 after the suppression of FoxO1 expression. c Western blotting analysis of the expression of p-PI3K, p-AKT, and TLR4 in LPS-stimulated primary microglia after transfection with FoxO1 siRNA and treated or not treated with baicalin. The levels of p-PI3K, p-AKT, and TLR4 were quantified. Data were normalized to the medium group. $\mathbf{d}$ The levels of IL-1 $\beta, I L-6$, and TNF-a in the cell culture supernatants of LPS-stimulated primary microglia were determined by ELISA after transfection with FoxO1 siRNA. All of the results are presented as means \pm SEM. ${ }^{\#} p<0.05,{ }^{\# \#} p<0.01$ vs. medium; ${ }^{*} p<0.05,{ }^{* *} p<0.01$ vs. LPS; ${ }^{\&} p<0.05,{ }^{\& \&} p<0.01$ vs. LPS + BA60; ${ }^{\$ \$ \$} p<0.005$ vs. LPS

role in the phosphorylation of PI3K and AKT and inhibit PI3K and AKT phosphorylation. Then, the total level of $\mathrm{p}$-PI3K and p-AKT is decreased.

FoxO1, which is a member of the forkhead transcription factor forkhead box protein $\mathrm{O}$ (FoxO) family, has important functions in cellular processes including DNA repair, cell cycle control, stress resistance, apoptosis, and cell metabolism $[65,66]$. FoxO1 is also a transcriptional regulator of TLR4 and its pro-inflammatory pathway by binding to multiple enhancer-like elements within the
TLR4 gene itself as well as to sites in several TLR4 signaling pathways [23]. Previous research has demonstrated that FoxO1 promotes the expression of IL-6, TNF- $\alpha$, and IL- $1 \beta$ in LPS-induced macrophages by directly transactivating the TLR4 gene and then upregulating the TLR4 gene expression [23]. Moreover, the activity of FoxO1 was mediated via phosphorylation by PI3K/AKT. The phosphorylation of FoxO1 by PI3K/ AKT was able to reduce its transactivation potential, which leads to the blockage of DNA binding, nuclear 

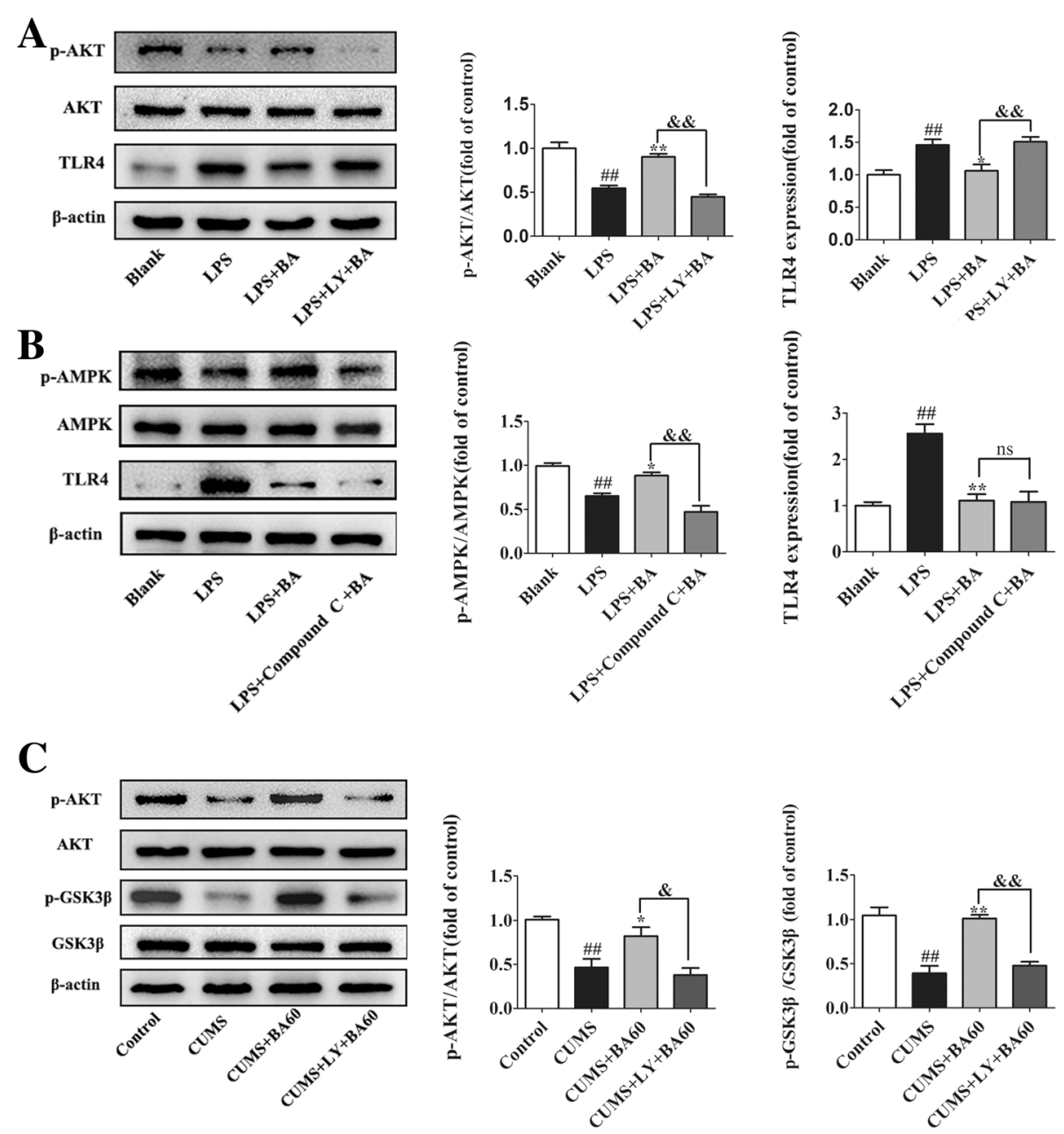

Fig. 10 A discussion of the potential targets of baicalin. a, b BV2 cells were pretreated with LY $292002(10 \mu M)$ and compound C (20 $\mu M)$ and coincubated with baicalin $(100 \mu \mathrm{M})$ for $1 \mathrm{~h}$ and then stimulated with LPS $(1 \mu \mathrm{g} / \mathrm{ml})$ for $24 \mathrm{~h}$. Western blot was performed to detect the protein levels of p-AKT, TLR4 $(\mathbf{a}, n=4), \mathrm{p}-\mathrm{AMPK}$, and TLR4 $(\mathbf{b}, n=4)$, and a statistical analysis was conducted. Data were normalized to the blank group and presented as means \pm SEM. ${ }^{*} p<0.05,{ }^{* *} p<0.01$ vs. blank; ${ }^{\#} p<0.05,{ }^{\# \#} p<0.01$ vs. LPS; ${ }^{\&} p<0.05,{ }^{\&}{ }^{\&} p<0.01$ vs. LPS+BA60. c Western blot was performed to measure the levels of p-AKT and p-GSK3 $\beta$ in the hippocampus of CUMS mice after treatment with LY294002 and baicalin ( $n=4)$. Data are presented as means \pm SEM. ${ }^{*} p<0.05,{ }^{* *} p<0.01$ vs. control; ${ }^{\#} p<0.05,{ }^{\# \#} p<0.01$ vs. CUMS; ${ }^{*} p<0.05,{ }^{\&} p<0.01$ vs. CUMS+BA60

exclusion, and subsequent sequestration into the cytoplasm and thus results in the inhibition of target gene expression [24, 67]. Consistent with previous findings, we demonstrated that the baicalin treatment greatly increased the expression of p-PI3K and p-AKT and then promoted the phosphorylation of FoxO1, contributed to FoxO1 nuclear exclusion, and finally resulted in attenuating the expression of TLR4 in the CUMS group mice as shown in Fig. 5 and Fig. 6. However, LY294002, which is a PI3K inhibitor, abolished the above effects of baicalin by inhibiting the activation of PI3K/AKT. Therefore, we depicted the molecular mechanisms in which baicalin might regulate the expression of TLR4 in CUMS mice via activation of the PI3K/AKT/FoxO1 pathway. Meanwhile, the effect of baicalin mediating the expression of TLR4 via the PI3K/AKT/FoxO1 pathway was also observed in LPS-induced inflammatory injury in BV2 cells and primary microglia cells (Fig. 7 and Fig. 8). Importantly, FoxO1, which is a transcriptional regulator, regulated TLR4 and its pro-inflammatory pathway in LPS-treated microglia cells and played a vital role in the inhibitory effect of baicalin on the LPS-simulated expression of TLR4 and TLR4-mediating neuroinflammation (Fig. 9).

Baicalin is a lipophilic flavonoid glycoside that can pass through the blood-brain barrier (BBB) and has therapeutic effects on the central nervous system $[19,68,69]$ including ameliorating depression [70] and cerebral ischemia $[18,20]$. We found that the anti-neuroinflammatory effect of baicalin in depressed mice was associated with downregulating TLR4 expression via activation of the PI3K/ AKT pathway. However, Li et al. found that baicalin 


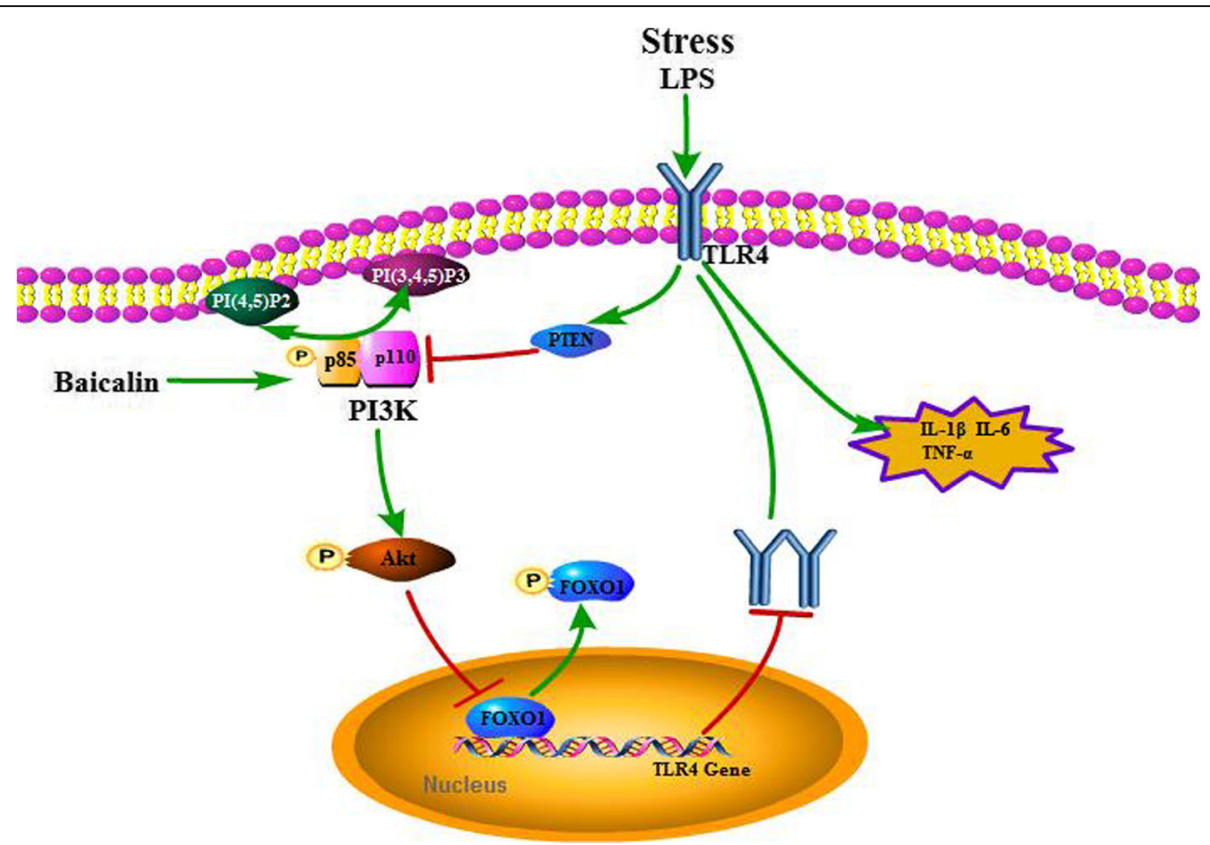

Fig. 11 A schematic illustration of the proposed mechanism for baicalin to restrain neuroinflammation

attenuated ischemia/reperfusion injury by regulating mitochondrial function in a manner dependent on AMPK [20]. AMPK involvement in downregulating TLR4 expression by baicalin had not been previously elucidated. To further this investigation, we employed LY294002 and compound $\mathrm{C}$ to specifically inhibit PI3K and AMPK in baicalin-treated BV2 cells, respectively. As Fig. 10a and b shows, the effect of baicalin on LPS-stimulated TLR4 expression could be abolished by LY294002 rather than compound C. Hence, baicalin might attenuate ischemia/ reperfusion injury depending on AMPK, but downregulated the expression of TLR4 via PI3K/AKT instead of AMPK. Recently, Zhang et al. discovered that baicalin exerts neuroprotective effects via inhibiting the activation of GSK3 $\beta$ in a rat model of depression [71]. There is evidence suggesting that glycogen synthase kinase-3 (GSK3) $\beta$ is a downstream molecule of PI3K/AKT [72], and the activation of PI3K/AKT results in the increase of p-GSK3 $\beta$ and then inhibits the activation of GSK3 $\beta$. We also investigated this molecular mechanism in CUMS mice as described in Fig. 10c. CUMS exposure repressed the phosphorylation of AKT $(p<0.01$, $F[3,12]=12.84)$ and $\operatorname{GSK} 3 \beta(p<0.01, F[3,12]=24.34)$ in mice, and the administration of baicalin greatly elevated the expression of $\mathrm{p}$-AKT and p-GSK3 $\beta$. LY294002 not only inhibits the phosphorylation of AKT, but also inhibits the phosphorylation of GSK3 $\beta$, which was consistent with a previous study showing that baicalin could inhibit the activation of GSK3 $\beta$. Interestingly, we also determined that baicalin inhibits the activation of GSK3 $\beta$ via the PI3K/AKT pathway.

\section{Conclusions}

The proposed pathway is shown in Fig. 11, and we demonstrated that baicalin could improve depressive-like behavior and ameliorate neuroinflammation in a CUMS and LPS-induced mice model of depression via the inhibition of pernicious overexpression of TLR4 via the $\mathrm{PI} 3 \mathrm{~K} / \mathrm{AKT} / \mathrm{FoxO} 1$ pathway. In addition, our results elucidated the underlying mechanisms of baicalin on the regulation of TLR4 expression in CUMS mice for the first time.

\section{Additional file}

Additional file 1: The details of primary mouse microglial cell culture. Figure S1. Iba-1 immunostaining images and morphology pictures of primary microglias isolated using mild trypsinization. Scale bar $=50 \mu \mathrm{m}$ (TIF 7.29 mb). Figure S2. Iba-1 immunostaining images and morphology pictures of primary microglia isolated using shaking. The scale bar $=50 \mu \mathrm{m}$ and arrows refer to the enlarged round cell body. (ZIP 24043 kb)

\section{Abbreviations}

AKT: Protein kinase B; AMPK: 5' adenosine monophosphate-activated protein kinase; CSF: Cerebrospinal fluid; CUMS: Chronic unpredictable mild stress; DMEM: Dulbecco's modified Eagle's medium; DMEM/F12: Dulbecco's modified Eagle's medium nutrient mixture F-12; ELISA: Enzyme-linked immunosorbent assay; FBS: Fetal bovine serum; Flu: Fluoxetine;

FoxO1: Forkhead box O1; FST: Forced swimming test; GSK3 $\beta$ : Glycogen synthase kinase-3 (GSK3) $\beta$; Iba1: lonized calcium-binding adapter molecule 1; IL-1 $\beta$ : Interleukin-1 beta; IL-6: Interleukin-6; LPS: Lipopolysaccharide; MDD: Major depressive disorder; OFT: Open field test; PBS: Phosphate buffer saline; PI3K: Phosphatidylinositol 3-kinase; PIP2: Phosphatidylinositol 3,4bisphosphate (PI (3,4)P2); PIP3: Phosphatidylinositol (3,4,5)-trisphosphate (PI(3,4,5)P3); SPT: Sucrose preference test; TLR4: Toll-like receptor 4; TNFa: Tumor necrosis factor alpha; TST: Tail suspension test 


\section{Acknowledgments}

We thank the staff members of our team for their cooperation in this work and Xue-Yang Deng for the assistance with this manuscript.

\section{Funding}

This work was supported by the National Natural Science Foundation of China (No. 81573701, No. 81703735), Chinese Postdoctoral Science Foundation (2017 M611958), Priority Academic Program Development of Jiangsu Higher Education Institutions (PAPD) and China Pharmaceutical University Undergraduate Training Programs for Innovation and Entrepreneurship (201810316154).

\section{Availability of data and materials}

Please contact the author for data requests.

\section{Authors' contributions}

L-TG designed this research. L-TG, S-QW, JS, L-XX, Z-YJ, R-YZ, and Q-WZ conducted the animal experiments. L-TG, S-QW, and JS performed the cellular experiments. L-TG wrote the manuscript. Z-QM, X-YD, and S-PM revised the manuscript. All authors read and approved the final manuscript.

\section{Ethics approval and consent to participate}

All animal use procedures were strictly performed in accordance with the Provision and General Recommendation of Chinese Experimental Animals Administration Legislation and were approved by the Science and Technology Department of Jiangsu Province.

\section{Consent for publication}

Not applicable.

\section{Competing interests}

The authors declare that they have no competing interests.

\section{Publisher's Note}

Springer Nature remains neutral with regard to jurisdictional claims in published maps and institutional affiliations.

\section{Author details}

'Department of Pharmacology of Chinese Materia Medica, China Pharmaceutical University, Tongjiaxiang 24, Nanjing 210009, Jiangsu, People's Republic of China. ${ }^{2}$ Qinba Traditional Chinese Medicine Resources Research and Development Center, AnKang University, AnKang 725000, People's Republic of China.

\section{Received: 29 September 2018 Accepted: 1 April 2019}

\section{Published online: 08 May 2019}

\section{References}

1. Franklin T, Xu C, Duman RS. Depression and sterile inflammation: essential role of danger associated molecular patterns. Brain Behavior and Immunity. 2018;72:2-13

2. Lamers F, Milaneschi Y, De Jonge P, Giltay EJ, Penninx BWJH. Metabolic and inflammatory markers: associations with individual depressive symptoms. Psychol Med. 2018;48:1102-10.

3. Slavich GM, Irwin MR. From stress to inflammation and major depressive disorder: a social signal transduction theory of depression. Psychiatr Bull. 2014:140:774-815

4. Raison $\mathrm{CL}$, Capuron L, Miller AH. Cytokines sing the blues: inflammation and the pathogenesis of depression. Trends Immunol. 2006;27:24-31.

5. Z Zhang C, Zhang YP, Li YY, Liu BP, Wang HY, Li KW, et al. Minocycline ameliorates depressive behaviors and neuro-immune dysfunction induced by chronic unpredictable mild stress in the rat. Behav Brain Res. 2019;356: 348-57

6. Jiang $\mathrm{H}$, Wang Z, Wang Y, Xie K, Zhang Q, Luan Q, et al. Antidepressant-like effects of curcumin in chronic mild stress of rats: involvement of its antiinflammatory action. Prog Neuro-Psychopharmacol Biol Psychiatry. 2013;47: 33-9.

7. Wright CE, Strike PC, Brydon L, Steptoe A. Acute inflammation and negative mood: mediation by cytokine activation. Brain Behav Immun. 2005;19:345-50.
8. Köhler CA, Freitas TH, Maes M, de Andrade NQ, Liu CS, Fernandes BS, et al. Peripheral cytokine and chemokine alterations in depression: a metaanalysis of 82 studies. Acta Psychiatr Scand. 2017;135:373-87.

9. Syed SA, Beurel E, Loewenstein DA, Lowell JA, Craighead WE, Dunlop BW, et al. Defective inflammatory pathways in never-treated depressed patients are associated with poor treatment response. Neuron. 2018;99:914-24.

10. Miller $\mathrm{AH}$, Raison $\mathrm{CL}$. The role of inflammation in depression: from evolutionary imperitive to modern treatment target. Nat Rev Immunol. 2016;16:22-34

11. Fleshner M, Frank M, Maier SF. Danger signals and inflammasomes: stressevoked sterile inflammation in mood disorders. Neuropsychopharmacology. 2017:42:36-45.

12. Cheng $Y$, Pardo M, Armini RS, Martinez A, Mouhsine H, Zagury JF, et al. Stress-induced neuroinflammation is mediated by GSK3-dependent TLR4 signaling that promotes susceptibility to depression-like behavior. Brain Behav Immun. 2016:53:207-22.

13. Gárate I, García-Bueno B, Madrigal JL, Bravo L, Berrocoso E, Caso JR, et al. Origin and consequences of brain toll-like receptor 4 pathway stimulation in an experim ental model of depression. J Neuroinflammation. 2011. https://doi.org/10.1186/1742-2094-8-151.

14. Caso JR, Pradillo JM, Hurtado O, Leza JC, Moro MA, Lizasoain I. Toll-like receptor 4 is involved in subacute stress-induced neuroinflammation and in the worsening of experimental stroke. Stroke. 2008;39:1314-20.

15. Zhao D, Xu X, Pan L, Zhu W, Fu X, Guo L, et al. Pharmacologic activation of cholinergic alpha7 nicotinic receptors mitigates depressive-like behavior in a mouse model of chronic stress. J Neuroinflammation. 2017;14:234.

16. Gárate I, García-Bueno B, Madrigal JLM, Caso JR, Alou L, Gómez-Lus ML, et al. Toll-like 4 receptor inhibitor TAK-242 decreases neuroinflammation in rat brain frontal cortex after stress. J Neuroinflammation. 2014;11:8.

17. Wang $Y, X$ J J, Liu Y, Li Z, Li X. TLR4-NF-kB signal involved in depressive-like behaviors and cytokine expression of frontal cortex and hippocampus in stressed C57BL/6 and ob/ob mice. Neural Plast. 2018. https://doi.org/10. 1155/2018/7254016

18. Chen W, Liang W, Huang $X$. The effects of baicalin and baicalein on cerebral ischemia: a review. Aging Dis. 2017:8:850-67.

19. Liang R, Han RM, Fu LM, Ai XC, Zhang JP, Skibsted LH. Baicalin in radical scavenging and its synergistic effect with $\beta$-carotene in antilipoxidation. J Agric Food Chem. 2009;57:7118-24.

20. Li S, Sun X, Xu L, Sun R, Ma Z, Deng X, et al. Baicalin attenuates in vivo and in vitro hyperglycemia-exacerbated ischemia/reperfusion injury by regulating mitochondrial function in a manner dependent on AMPK. Eur J Pharmacolol. 2017;815:118-26.

21. Zuo D, Lin L, Liu Y, Wang C, Xu JSF, et al. Baicalin attenuates ketamineinduced neurotoxicity in the developing rats: involvement of PI3K/Akt and CREB/BDNF/BCl-2 pathways. Neurotox Res. 2016;30:159-72.

22. Zhou ZQ, Li YL, Ao ZB, Wen ZL, Chen QW, Huang ZG, et al. Baicalin protects neonatal rat brains against hypoxic- ischemic injury by upregulating glutamate transporter 1 via the phosphoinositide 3-kinase/protein kinase B signaling pathway. Neural Regen Res. 2017;12:1625-31.

23. Fan W, Morinaga H, Kim JJ, Bae E, Spann NJ, Heinz S, et al. FoxO1 regulates Tru inflammatory pathway signalling in macrophages. EMBO J. 2010;29:4223-36.

24. Wagatsuma A, Shiozuka M, Takayama Y, Hoshino T, Mabuchi K, Matsuda R. Effects of ageing on expression of the muscle-specific E3 ubiquitin ligases and Akt-dependent regulation of Foxo transcription factors in skeletal muscle. Mol Cell Biochem. 2016:412:59-72.

25. Cheng P, Wang T, Li W, Muhammad I, Wang H, Sun X, et al. Baicalin alleviates lipopolysaccharide-induced liver inflammation in chicken by suppressing TLR4-mediated NF-KB pathway. Front Pharmacol. 2017:8:547.

26. Sun JY, Li DL, Dong Y, Zhu CH, Liu J, Li JD, et al. Baicalin inhibits toll-like receptor $2 / 4$ expression and downstream signaling in rat experimental periodontitis. Int Immunopharmacol. 2016:36:86-93.

27. Li Z, Xia X, Zhang S, Zhang A, Bo W, Zhou R. Up-regulation of toll-like receptor 4 was suppressed by emodin and baicalin in the setting of acute pancreatitis. Biomed Pharmacother. 2009:63:120-8.

28. Cui $L$, Feng $L$, Zhang $Z$ H, Jia XB. The anti-inflammation effect of baicalin on experimental colitis through inhibiting TLR4/NF-KB pathway activation. Int Immunopharmacol. 2014;23:294-303.

29. Thakare VN, Patil RR, Oswal RJ, Dhakane VD, Aswar MK, Patel BM Therapeutic potential of silymarin in chronic unpredictable mild stress induced depressive-like behavior in mice. J Psychopharmacol. 2018; $32: 223-35$ 
30. Song Y, Sun R, Ji Z, Li X, Fu Q, Ma S. Perilla aldehyde attenuates CUMSinduced depressive-like behaviors via regulating TXNIP/TRX/NLRP3 pathway in rats. Life Sci. 2018;206:117-24.

31. Pan $Y$, Chen XY, Zhang QY, Kong L. Microglial NLRP3 inflammasome activation mediates IL-1 b -related inflammation in prefrontal cortex of depressive rats. Brain Behav Immun. 2014:41:90-100.

32. Song MT, Ruan J, Zhang RY, Deng J, Ma ZQ, Ma SP. Astragaloside IV ameliorates neuroinflammation- induced depressive-like behaviors in mice via the PPARY/NF-kB/NLRP3 inflammasome axis. Acta Pharmacol Sin. 2018; 39:1559-70.

33. Yu HY, Yin ZJ, Yang SJ, Ma SP. Baicalin reverse AMPA receptor expression and neuron apoptosis in chronic unpredictable mild stress rats. Biochem Biophys Res Commun. 2014:451:467-72.

34. Liu X, Liu C. Baicalin ameliorates chronic unpredictable mild stress-induced depressive behavior: involving the inhibition of NLRP3 inflammasome activation in rat prefrontal cortex. Int Immunopharmacol. 2017:48:30-4.

35. Kim S-N, Kim S-T, Doo A-R, Park J-Y, Moon W. Phosphatidylinositol 3-kinase/ Akt signaling pathway mediates acupuncture-induced dopaminergic neuron protection and motor function improvement in a mouse model of Parkinson's disease. Int J Neurosci. 2011;121:562-9.

36. Shao S, Wang GL, Raymond C, Deng XH, Zhu XL, Wang Dl, et al. Activation of sonic hedgehog signal by purmorphamine, in a mouse model of Parkinson's disease, protects dopaminergic neurons and attenuates inflammatory response by mediating PI3K/AKt signaling pathway. Mol Med Rep. 2017;16:1269-77.

37. Fernández-calle R, Vicente-rodríguez M, Gramage E, Pita J, Pérez-garcía C, Ferrer-alcón $M$, et al. Pleiotrophin regulates microglia-mediated neuroinflammation. J Neuroinflammation. 2017. https://doi.org/10.1186/ s12974-017-0823-8.

38. Fellner A, Barhum Y, Angel A, Perets N, Steiner I, Offen D, et al. Toll-like receptor-4 inhibitor TAK-242 attenuates motor dysfunction and spinal cord pathology in an amyotrophic lateral sclerosis mouse model. Int J Mol Sci. 2017;18:1666.

39. Walker AK, Wing EE, Banks WA, Dantzer R. Leucine competes with kynurenine for blood-to-brain transport and prevents lipopolysaccharideinduced depression-like behavior in mice. Mol Psychiatry. 2018. https://doi. org/10.1038/s41380-018-0076-7.

40. Bhattacharya A, Lord B, Grigoleit JS, He Y, Fraser I, Campbell SN, et al. Neuropsychopharmacology of JNJ-55308942: evaluation of a clinical candidate targeting P2X7 ion channels in animal models of neuroinflammation and anhedonia. Neuropsychopharmacology. 2018 https://doi.org/10.1038/s41386-018-0141-6.

41. O'Connor JC, Lawson MA, André C, Moreau M, Lestage J, Castanon N, et al. Lipopolysaccharide-induced depressive-like behavior is mediated by indoleamine 2,3-dioxygenase activation in mice. Mol Psychiatry. 2009;14: $511-22$

42. Zhu XL, Chen JJ, Han F, Pan C, Zhuang TT, Cai YF, et al. Novel antidepressant effects of Paeonol alleviate neuronal injury with concomitant alterations in BDNF, Rac1 and RhoA levels in chronic unpredictable mild stress rats. Psychopharmacology. 2018;235:2177-91.

43. Gu M, Li Y, Tang H, Zhang C, Li W, Zhang Y, et al. Endogenous omega (n)-3 fatty acids in fat-1 mice attenuated depression-like behavior, imbalance between microglial M1 and M2 phenotypes, and dysfunction of neurotrophins induced by lipopolysaccharide administration. Nutrients. 2018. https://doi.org/10.3390/nu10101351.

44. Xu Y, Sheng H, Bao Q, Wang Y, Lu J, Ni X. NLRP3 inflammasome activation mediates estrogen deficiency-induced depression- and anxiety-like behavior and hippocampal inflammation in mice. Brain Behav Immun. 2016;56:175-86.

45. Oh DR, Yoo JS, Kim Y, Kang H, Lee H, Lm SJ, et al. Vaccinium bracteatum leaf extract reverses chronic restraint stress-induced depression-like behavior in mice: regulation of hypothalamic-pituitary-adrenal axis, serotonin turnover systems, and ERK/Akt phosphorylation. Front Pharmacol. 2018;9:604.

46. Zhang H, Li K, Chen HS, Gao SQ, Xia ZX, Zhang JT, et al. Dorsal raphe projection inhibits the excitatory inputs on lateral habenula and alleviates depressive behaviors in rats. Brain Struct Funct. 2018;223:2243-58.

47. Hu X, Dong Y, Jin X, Zhang C, Zhang T, Zhao J. The novel and potent antidepressive action of triptolide and its influences on hippocampal neuroinflammation in a rat model of depression comorbidity of chronic pain. Brain Behav Immun. 2017;64:180-94.

48. Xu K, Liu Z, Wang L, Wu G, Liu T. Influence of hippocampal low-frequency stimulation on GABAA R a1, ICER and BNDF expression level in brain tissues of amygdala-kindled drug-resistant temporal lobe epileptic rats. Brain Res. 2018;1698:195-203.

49. Santiago A, Soares LM, Schepers M, Milani H, Vanmierlo T, Prickaerts J, et al. Roflumilast promotes memory recovery and attenuates white matter injury in aged rats subjected to chronic cerebral hypoperfusion. Neuropharmacology. 2018;13:360-70.

50. Rehman SU, Ali T, Alam SI, Ullah R, Zeb A, Lee KW, et al. Ferulic acid rescues LPS-induced neurotoxicity via modulation of the TLR4 receptor in the mouse Hippocampus. Mol Neurobiol. 2018. https://doi.org/10.1007/s12035018-1280-9.

51. Chen $L$, Tao $Y$, Jiang $Y$. Apelin activates the expression of inflammatory cytokines in microglial BV2 cells via PI-3K/Akt and MEK/Erk pathways. Sci China Life Sci. 2015;58:531-40.

52. Saura J, Tusell JM, Serratosa J. High-yield isolation of murine microglia by mild trypsinization. Glia. 2003;44:183-9. https://doi.org/10.1002/glia.10274.

53. Lin L, Desai R, Wang $X$, Lo EH, Xing C. Characteristics of primary rat microglia isolated from mixed cultures using two different methods. $J$ Neuroinflammation. 2017;14. https://doi.org/10.1186/s12974-017-0877-7.

54. Matsunaga N, Tsuchimori N, Matsumoto T, li M. TAK-242 (resatorvid), a small-molecule inhibitor of toll-like receptor (TLR) 4 signaling, binds selectively to TLR4 and interferes with interactions between TLR4 and its adaptor molecules. Mol Pharmacol. 2011;79:34-41.

55. Dong H, Zhang X, Dai X, Lu S, Gui B, Jin W, et al. Lithium ameliorates lipopolysaccharide-induced microglial activation via inhibition of toll-like receptor 4 expression by activating the PI3K/Akt/FoxO1 pathway. J Neuroinflammation. 2014;11:140.

56. Kim SY, Jeong E, Joung SM, Lee JY. PI3K / Akt contributes to increased expression of toll-like receptor 4 in macrophages exposed to hypoxic stress. Biochem and Biophys Res Commun. 2012;419:466-71.

57. Yue N, Huang H, Zhu X, Han Q, Wang Y, Li B, et al. Activation of P2X7 receptor and NLRP3 inflammasome assembly in hippocampal glial cells mediates chronic stress-induced depressive-like behaviors. J Neuroinflammation. 2017;14:102.

58. Gárate I, Garcia-Bueno B, Madrigal JL, Caso JR, Alou L, Gomez-Lus ML, et al. Stress-induced Neuroinflammation : role of the toll-like receptor-4 pathway. Biol Psychiatry. 2013;73:32-43.

59. Zhao M, Zhou A, Xu L, Zhang X. The role of TLR4-mediated PTEN/PI3K/AKT/ NF-KB signaling pathway in neuroinflammation in hippocampal neurons. Neuroscience. 2014;269:93-101.

60. Zhou M, Zhang Y, Chen X, Zhu J, Du M, Zhou L, et al. PTEN - Foxo1 signaling triggers HMGB1-mediated innate immune responses in acute lung injury. Immunol Res. 2015;62:95-105.

61. Sonoda Y, Mukai H, Matsuo K, Takahashi M, Ono Y, Maeda K, et al. Accumulation of tumor-suppressor PTEN in Alzheimer neurofibrillary tangles. Neurosci Lett. 2010;471:20-4.

62. Maehama T, Dixon JE. The tumor suppressor, PTEN/MMAC1, dephosphorylates the lipid second messenger, phosphatidylinositol 3,4,5trisphosphate. J Biol Chem. 1998;29:13375-8.

63. Lee JO, Yang H, Georgescu MM, Di CA, Maehama T, Shi Y, et al. Crystal structure of the PTEN tumor suppressor: implications for its phosphoinositide phosphatase activity and membrane association. Cell. 1999;99:323-34.

64. Saponaro C, Cianciulli A, Calvello R, Dragone T, lacobazzi F, Panaro MA. The PI3K/Akt pathway is required for LPS activation of microglial cells. Immunopharmacol Immunotoxicol. 2012:34:858-65.

65. Furukawa-Hibi Y, Kobayashi Y, Chen C, Motoyama N. FOXO transcription factors in cell-cycle regulation and the response to oxidative stress. Antioxid Redox Signal. 2005;7:752-60.

66. Nakae J, Barr V, Accili D. Differential regulation of gene expression by insulin and IGF-1 receptors correlates with phosphorylation of a single amino acid residue in the forkhead transcription factor FKHR. EMBO J. 2000;19:989-96.

67. Brunet A, Bonni A, Zigmond MJ, Lin MZ, Juo P, Hu LS, et al. Akt promotes cell survival by phosphorylating and inhibiting a Forkhead transcription factor. Cell. 1999;96:857-68.

68. Fong SYK, Li C, Ho YC, Li R, Wang Q, Wong YC, et al. Brain uptake of bioactive flavones in Scutellariae Radix and its relationship to anxiolytic effect in mice. Mol Pharm. 2017;14:2908-16.

69. Huang $H$, Zhang $Y$, Yang $R$, Tang. Determination of baicalin in rat cerebrospinal fluid and blood using microdialysis coupled with ultraperformance liquid chromatography-tandem mass spectrometry. J Chromatogr B 2008;874:77-83. 
70. Zhang CY, Zeng MJ, Zhou LP, Li YQ, Zhao F, Shang Z, et al. Baicalin exerts neuroprotective effects via inhibiting activation of GSK3ß/NF-KB/NLRP3 signal pathway in a rat model of depression. Int Immunopharmacol. 2018; 64:175-82.

71. Deng S, Dai G, Chen S, Nie Z, Zhou J, Fang H. Dexamethasone induces osteoblast apoptosis through ROS-PI3K/AKT/GSK3 $\beta$ signaling pathway. Biomed Pharmacother. 2019;110:602-8.

72. Li H, Chen Y, Zhang J, Chen X, Li Z, Zhang L. Shikonin attenuates acetaminophen-induced hepatotoxicity by upregulation of Nrf2 through Akt/GSK3ß signaling. Molecules. 2018;24. https://doi.org/10.3390/ molecules 24010110

Ready to submit your research? Choose BMC and benefit from:

- fast, convenient online submission

- thorough peer review by experienced researchers in your field

- rapid publication on acceptance

- support for research data, including large and complex data types

- gold Open Access which fosters wider collaboration and increased citations

- maximum visibility for your research: over $100 \mathrm{M}$ website views per year

At $\mathrm{BMC}$, research is always in progress.

Learn more biomedcentral.com/submissions 